\title{
Shear stress-induced endothelial adrenomedullin signaling regulates vascular tone and blood pressure
}

\author{
Andras Iring, ${ }^{1}$ Young-June Jin, ${ }^{1}$ Julián Albarrán-Juárez, ${ }^{1}$ Mauro Siragusa, ${ }^{2,3}$ ShengPeng Wang, ${ }^{1,4}$ Péter T. Dancs, ${ }^{5}$ \\ Akiko Nakayama, ${ }^{1}$ Sarah Tonack, ${ }^{1}$ Min Chen, ${ }^{6}$ Carsten Künne, ${ }^{7}$ Anna M. Sokol, ${ }^{8}$ Stefan Günther, ${ }^{7}$ Alfredo Martínez, ${ }^{9}$ \\ Ingrid Fleming, ${ }^{2,3}$ Nina Wettschureck, ${ }^{1,3,10}$ Johannes Graumann, ${ }^{3,8}$ Lee S. Weinstein, ${ }^{6}$ and Stefan Offermanns ${ }^{1,3,10}$
}

'Department of Pharmacology, Max Planck Institute for Heart and Lung Research, Bad Nauheim, Germany. IInstitute for Vascular Signalling, Centre for Molecular Medicine, Goethe University, Frankfurt am Main, Germany. ${ }^{3}$ Cerman Centre for Cardiovascular Research (DZHK), Rhine-Main site, Frankfurt and Bad Nauheim, Germany. . ${ }^{4}$ Cardiovascular Research Center, School of Basic Medical Sciences, Xi'an Jiaotong University Health Science Center, Yanta District, Xi'an, China. Innstitute of Clinical Experimental Research, Semmelweis University, Budapest, Hungary. ${ }^{6}$ Metabolic Diseases Branch, National Institute of Diabetes and Digestive and Kidney Diseases, NIH, Bethesda, Maryland, USA. Bioinformatics and Deep Sequencing Platform and ${ }^{8}$ Scientific Service Group Biomolecular Mass Spectrometry, Max Planck Institute for Heart and Lung Research, Bad Nauheim, Germany. ${ }^{9}$ Oncology Area, Center for Biomedical Research of La Rioja (CIBIR), Logroño, Spain. ${ }^{10}$ Centre for Molecular Medicine, Medical Faculty, Goethe University, Frankfurt am Main, Germany.

\begin{abstract}
Hypertension is a primary risk factor for cardiovascular diseases including myocardial infarction and stroke. Major determinants of blood pressure are vasodilatory factors such as nitric oxide (NO) released from the endothelium under the influence of fluid shear stress exerted by the flowing blood. Several endothelial signaling processes mediating fluid shear stress-induced formation and release of vasodilatory factors have been described. It is, however, still poorly understood how fluid shear stress induces these endothelial responses. Here we show that the endothelial mechanosensitive cation channel PIEZO1 mediated fluid shear stress-induced release of adrenomedullin, which in turn activated its $\mathrm{G}_{\mathrm{s}}$-coupled receptor. The subsequent increase in CAMP levels promoted the phosphorylation of endothelial NO synthase (eNOS) at serine 633 through protein kinase $A$ (PKA), leading to the activation of the enzyme. This $G_{s} /$ PKA-mediated pathway synergized with the AKT-mediated pathways leading to eNOS phosphorylation at serine 1177. Mice with endothelium-specific deficiency of adrenomedullin, the adrenomedullin receptor, or $\mathrm{G} \alpha_{s}$ showed reduced flow-induced eNOS activation and vasodilation and developed hypertension. Our data identify fluid shear stress-induced PIEZO1 activation as a central regulator of endothelial adrenomedullin release and establish the adrenomedullin receptor and subsequent $\mathrm{G}_{\mathrm{s}}$-mediated formation of $\mathrm{cAMP}$ as a critical endothelial mechanosignaling pathway regulating basal endothelial NO formation, vascular tone, and blood pressure.
\end{abstract}

\section{Introduction}

Vascular tone and blood pressure are controlled by various autoregulatory functions of blood vessels. Central among them is the ability of the endothelium to sense fluid shear stress exerted by the flowing blood and to respond to it with the release of vasodilatory factors such as nitric oxide (NO) (1). Flow-induced vasorelaxation mediated by NO is important for the adaptation of vessel diameter to blood flow and also controls vascular tone and blood pressure (2). NO is generated by endothelial NO synthase (eNOS, or NOS3), the activity of which is increased by fluid shear stress through various mechanisms $(3-5)$. While an acute increase in flow results in a transient elevation of intracellular $\left[\mathrm{Ca}^{2+}\right]$ and the subsequent $\mathrm{Ca}^{2+} /$ calmodulin-dependent activation of eNOS $(3,6,7)$, a sustained flow-induced NO formation requires phosphorylation of the enzyme (3-5). One of the phosphorylation sites of human eNOS that is phosphorylated in response to flow is serine 1177 (serine 1179 in bovine eNOS),

Conflict of interest: The authors have declared that no conflict of interest exists. Copyright: () 2019, American Society for Clinical Investigation.

Submitted: August 13, 2018; Accepted: May 1, 2019; Published: June 17, 2019.

Reference information: J Clin Invest. 2019;129(7):2775-2791.

https://doi.org/10.1172/JCl123825. which can be phosphorylated by AKT and other kinases $(8,9)$. The activation of endothelial AKT by flow involves a mechanosensory complex consisting of PECAM-1, VE-cadherin, and VEGFR2/VEGFR3 (10-13). Recent data indicate that the endothelial mechanosensitive cation channel PIEZO1 is activated by fluid shear stress $(14,15)$ and mediates the flow-induced release of ATP from endothelial cells, which results in the activation of the $G_{q} / G_{11}$-coupled purinergic P2Y receptor (16). $\mathrm{G}_{\mathrm{q}} / \mathrm{G}_{11}$-mediated signaling in turn is required for flow-dependent activation of the PECAM-1/VE-cadherin/VEGFR mechanosensing complex, which results in stimulation of AKT activity and subsequent phosphorylation of eNOS at serine 1177, leading to increased NO formation (17).

In addition to serine 1177, serine 633 (serine 635 in bovine eNOS) has been shown to be phosphorylated by fluid shear stress $(3,18)$. Initial reports comparing the functional consequences of human eNOS phosphorylation at serine 1177 versus serine 633 concluded that phosphorylation of serine 1177 played the major role in the regulation of NO formation, largely because phosphorylation on serine 633 appeared to be without functional consequences or could not be detected $(8,9)$. Later work, however, indicated that phosphorylation of human and bovine eNOS on serine 633 and 635, respectively, increases eNOS enzymatic activity 
(19-21) and that phosphorylation of bovine eNOS at both of the serine residues acts synergistically to activate the enzyme (22). Several reports indicate that the cAMP-activated protein kinase (PKA) phosphorylates bovine and human eNOS at serine 635/633 $(19,23,24)$ and that PKA mediates fluid shear stress-induced phosphorylation of bovine eNOS at serine 635 (24). However, the time course of flow-induced eNOS phosphorylation at serine 635 was significantly slower than that of phosphorylation at serine 1179 (24). These mechanotransduction processes have been studied in vitro using isolated endothelial cells, but their relevance in vivo is still unclear, and the mechanisms underlying the flow-induced activation of PKA and subsequent phosphorylation of eNOS on serine 633/635 have remained elusive.

Here we show that the mechanosensitive cation channel PIEZO1 mediates the fluid shear stress-induced release of adrenomedullin from endothelial cells. Adrenomedullin then induces the activation of the $\mathrm{G}_{\mathrm{s}}$-coupled endothelial adrenomedullin receptor to increase intracellular cAMP levels. Through activation of PKA, this signaling cascade couples fluid shear stress to eNOS phosphorylation at serine 633/635 and thereby promotes NO formation and regulates vascular tone and blood pressure.

\section{Results}

Fluid shear stress induces $G_{s}$-dependent eNOS phosphorylation in vitro. In initial experiments we confirmed that the specific myristoylated PKA inhibitor peptide PKI $(25,26)$ blocks flow-induced eNOS phosphorylation in bovine aortic endothelial cells (BAECs) at serine 635, but not phosphorylation of serine 1179 (Figure 1A). Since cAMP is the major regulator of PKA (27), we determined intracellular cAMP levels in BAECs kept under static conditions or after exposure to fluid shear stress for up to 30 minutes (Figure 1B). As reported previously in human umbilical vein endothelial cells (HUVECs) (28), fluid shear stress induced an increase in cAMP levels in BAECs after several minutes (Figure 1B). Since the heterotrimeric $G$ protein $G_{s}$ couples multiple $G$ protein-coupled receptors (GPCRs) in a stimulatory fashion to adenylyl cyclase to produce cAMP $(27,29,30)$, we tested the role of $G_{s}$ in fluid shear stress-induced endothelial responses. The siRNA-mediated knockdown of the $\alpha$ subunit of $G, G \alpha_{s}$ (Supplemental Figure 1A; supplemental material available online with this article; https:// doi.org/10.1172/JCI123825DS1), prevented the shear stressinduced increase in cAMP (Figure 1B). Knockdown of $G \alpha_{s}$ also reduced cAMP increases induced by the $\beta$-adrenoceptor agonist isoproterenol but had no effect on forskolin-induced effects (Supplemental Figure 1B).

Using mass spectrometry-based phosphoproteomic analysis of BAECs, we found 7 serine/threonine residues of bovine eNOS to be phosphorylated. Of those, serine 635, threonine 1177 , and serine 1179 as well as serine 1181 showed increased phosphorylation in response to 30 minutes of laminar flow (Figure 1C). Only flow-induced phosphorylation of serine 635 was sensitive to knockdown of $\mathrm{G}_{\mathrm{s}}$ (Figure 1C). This finding was validated by Western blot analysis showing that the fluid shear stress-induced phosphorylation of eNOS on serine 635, which developed relatively slowly after induction of flow, was almost completely prevented by knockdown of $\mathrm{G} \alpha_{\mathrm{s}}$. In contrast, flow-induced eNOS phosphorylation on serine 1179 and phosphorylation of AKT, which occurred much faster after induction of flow, were unaffected (Figure 1, D and E). Similar results were obtained using human aortic endothelial cells (HAECs) (Supplemental Figure 1C). Activation of the $\mathrm{G}_{\mathrm{s}}$-coupled $\beta$-adrenergic receptor by isoproterenol induced eNOS phosphorylation at serine 635 but not at serine 1179, and the effect of isoproterenol was blocked after knockdown of $\mathrm{G} \alpha_{\mathrm{s}}$ (Figure $1 \mathrm{~F}$ ). In contrast, VEGF-induced phosphorylation of eNOS and AKT was insensitive to $G \alpha_{\mathrm{s}}$ knockdown (Figure $1 \mathrm{G}$ and Supplemental Figure 1D), indicating that the cells had not lost the ability to respond with eNOS phosphorylation to $\mathrm{G}_{\mathrm{s}}$-independent stimuli.

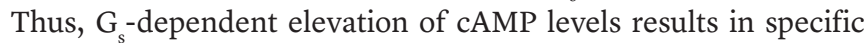
phosphorylation of eNOS at serine 635.

$G_{s}$ and $G_{q} / G_{11}$ mediate flow-induced eNOS activation in an additive manner. In contrast to knockdown of $\mathrm{G} \alpha$, knockdown of $\mathrm{G} \alpha_{\mathrm{q}} / \mathrm{G} \alpha_{11}$ had no effect on flow-induced eNOS phosphorylation at serine 635 but, as described earlier, blocked flow-induced eNOS phosphorylation at serine 1179 as well as AKT phosphorylation (ref. 17 and Figure 2A). Within the first 5 minutes after onset of flow, knockdown of $\mathrm{G} \alpha_{\mathrm{s}}$ had no effect on flow-induced increases in NO levels in cellular supernatants as determined by measurement of nitrite/nitrate levels (Figure 2B). With increasing flow times, however, the relative contribution of $\mathrm{G}_{\mathrm{s}}$-mediated signaling increased, and 60 minutes after onset of flow, knockdown of $\mathrm{G} \alpha_{\mathrm{s}}$ expression reduced flow-induced $\mathrm{NO}$ formation by more than 50\% (Figure 2B and Supplemental Figure 1E). Similar results were obtained when HAECs were used (Supplemental Figure 1F). In contrast, knockdown of $G \alpha_{q} / G \alpha_{11}$ expression in endothelial cells strongly affected flow-induced NO formation already at early flow times, but the effect decreased at flow times longer than 30 minutes (Figure 2B). Thus, NO formation in response to acute onset of flow initially requires $G_{q} / G_{11}$-mediated signaling; however, at longer times of flow, flow-induced NO formation also depends on $\mathrm{G}_{\mathrm{s}}$-mediated signaling. This observation correlates with the delayed onset of $\mathrm{G}_{\mathrm{s}}$-mediated eNOS phosphorylation at serine 635 (Figure 1, D and E). The effects of $G \alpha_{q} / G \alpha_{11}$ and $G \alpha_{s}$ knockdown on flow-induced NO formation were additive (Figure 2B). Consistent with this, flow-induced NO formation in HAECs was abrogated after knockdown of eNOS and partially rescued by re-expression of the human eNOS phospho-site mutants S633A and S1177A, in comparison with cells in which WT eNOS was re-expressed (Figure 2C and Supplemental Figure 1G).

Endothelial $G_{s}$ deficiency results in hypertension and loss of flow-induced vasodilation. To test whether $\mathrm{G}_{\mathrm{s}}$-mediated endothelial flow sensing is physiologically relevant, we generated mice with tamoxifen-inducible, endothelium-specific $\mathrm{G} \alpha_{\mathrm{s}}$ deficiency $\left(\right.$ Tek-CreER ${ }^{T 2}$;Gnas ${ }^{f / f l}$, herein referred to as EC-G $\left.\alpha_{s}-\mathrm{KO}\right)$ (Supplemental Figure $1 \mathrm{H})$. Aortic segments from induced $\mathrm{EC}-\mathrm{G} \alpha_{\mathrm{s}}-\mathrm{KO}$ mice showed normal contractile responses to phenylephrine, and precontracted vessels responded to acetylcholine similarly to vessels from WT mice (Figure 3, A and B). This indicates that the contractile and vasodilatory functions of arteries lacking endothelial $G_{s}$ were unaffected. However, endothelial loss of $G_{s}$ resulted in strong reduction of flow-induced dilation of precontracted mesenteric vessels (Figure $3 \mathrm{C}$ ).

Since vascular tone and blood pressure are influenced by flow-induced vasodilation $(31,32)$, we tested the effect of an acute 
A
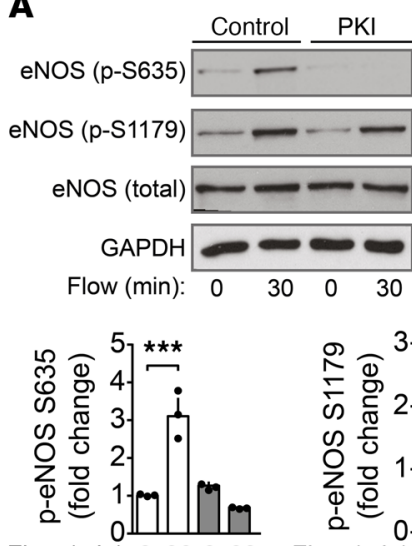

Flow (min): 030030<smiles>C1C[C@H]2C[C@H]1C2</smiles>

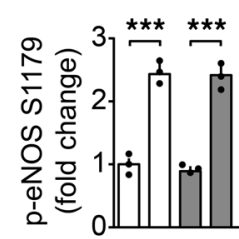

Flow (min): 030030

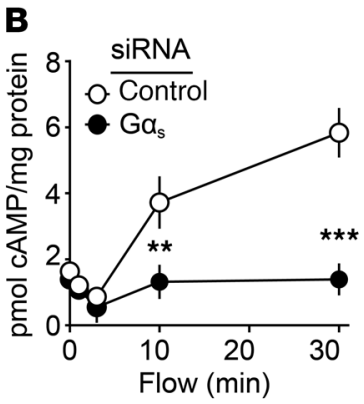

C

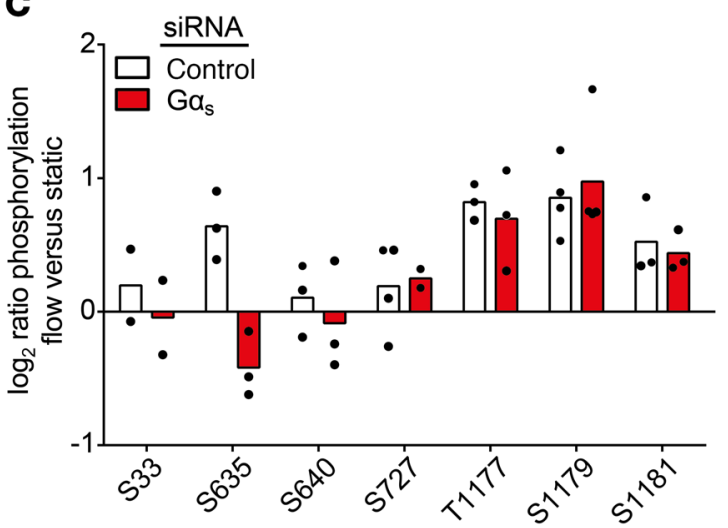

D

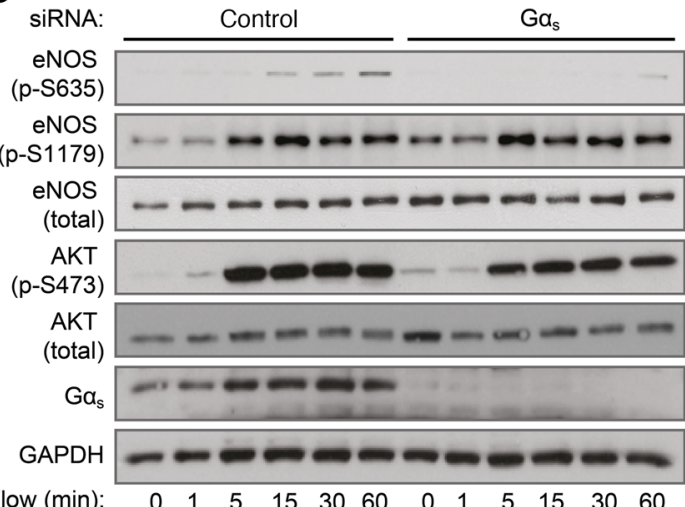

E
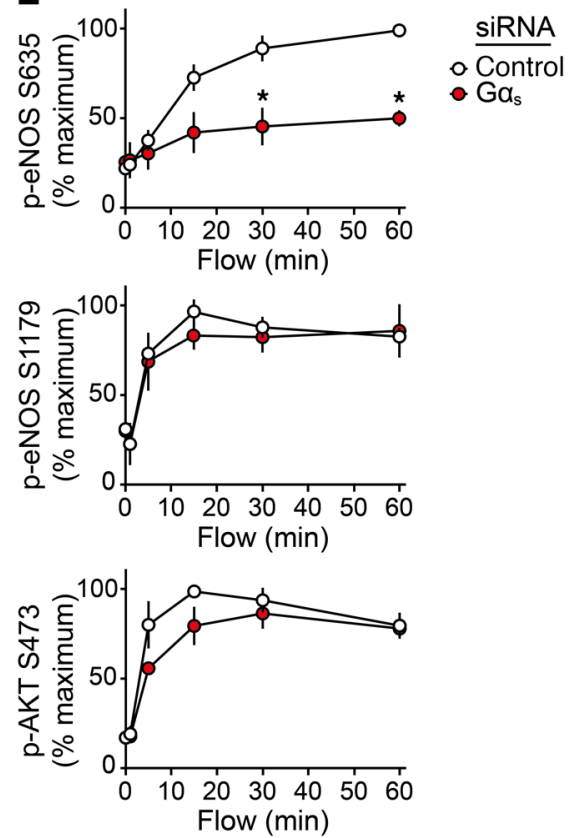

$\mathbf{F}$
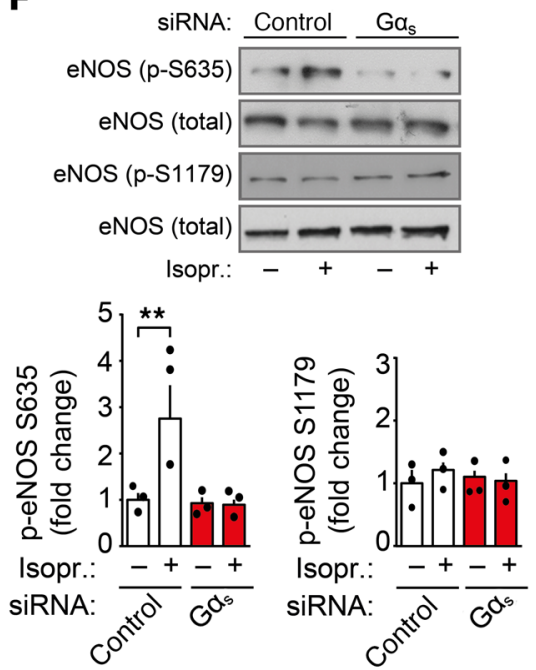

G
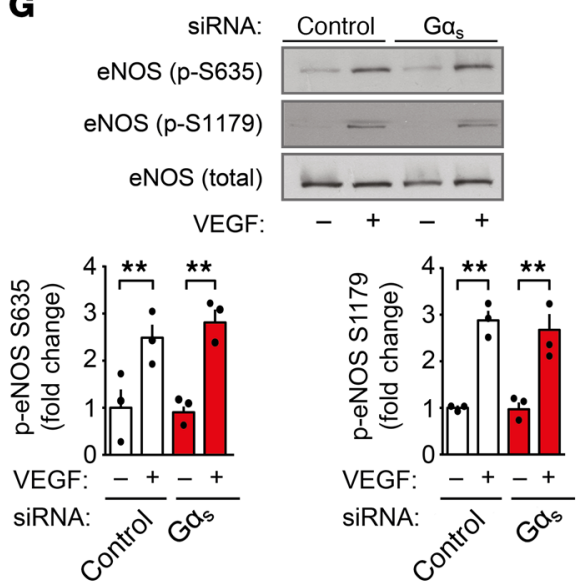

Figure 1. $G_{s}$ mediates flow-induced CAMP formation and specific eNOS phosphorylation at serine 635 in BAECs. (A) BAECs were pretreated with the PKA inhibitor PKI $(1 \mu \mathrm{M})$ or solvent (control) for 20 minutes and were then kept under static conditions or were exposed to flow $\left(15 \mathrm{dyn} / \mathrm{cm}^{2}\right)$ for 30 minutes. Total and phosphorylated eNOS was determined by immunoblotting. Graphs show the densitometric evaluation $(n=3)$. (B-G) BAECs were transfected with scrambled (control) siRNA or siRNA directed against $\mathrm{C} \alpha$ s as indicated and were exposed to flow for 30 minutes or the indicated time periods $\left(15 \mathrm{dyn} / \mathrm{cm}^{2}\right.$ in B-E) or were incubated with isoproterenol (Isopr., $10 \mu \mathrm{M}, 10$ minutes) or VEGF $(50 \mathrm{ng} / \mathrm{ml}, 10$ minutes) (F and $\mathbf{G}$ ). (B) Intracellular CAMP levels were determined ( $n=4$, control; $n=6, G \alpha_{s}$ ). (C) Phosphorylation of various eNOS sites was determined by LC-MS/MS under static conditions and after application of shear stress

$\left(15 \mathrm{dyn} / \mathrm{cm}^{2}\right)$ for 30 minutes. Intensity values of the phoshpo-sites shown were normalized to eNOS intensity and thus protein abundance in the proteome. Bar diagrams show the fold change of the corrected ratios of flow versus static conditions $(n=2-4)$.

Logarithmization of the $y$ axis emphasizes the directional deviation from the static condition. (D-G) Total and phosphorylated eNOS and AKT were analyzed by immunoblotting. Graphs and bar diagrams show the densitometric evaluation $(n=3)$. Analysis of $\mathrm{G} \alpha$ s and GAPDH expression served as control (D). Data represent the mean $\pm \mathrm{SEM} ;{ }^{*} P \leq 0.05,{ }^{* *} P \leq 0.01$, ${ }^{* * *} P \leq 0.001,1$-way ANOVA with Tukey's post hoc test (A, F, and $\mathbf{G})$, 2-way ANOVA with Bonferroni's post hoc test (B and $\mathbf{E}$ ). 
A
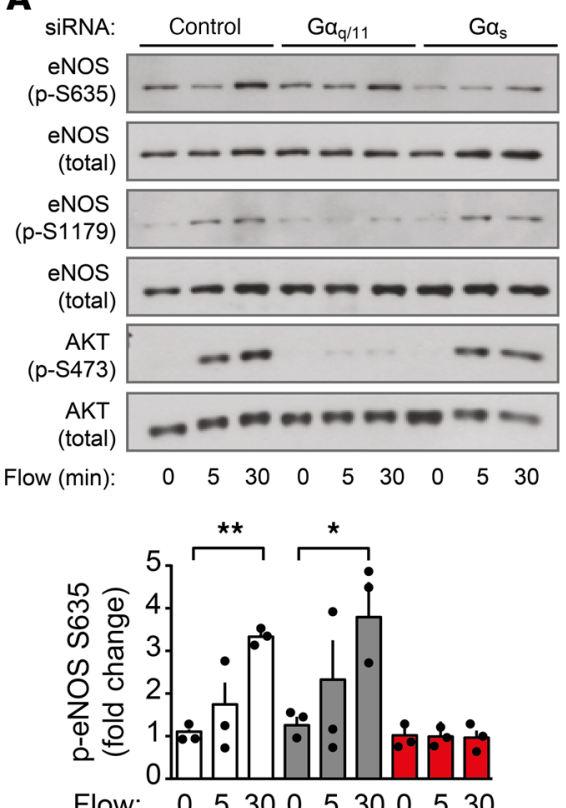

Flow: $0 \begin{array}{llllllll} & 30 & 0 & 5 & 30 & 0 & 5 & 30\end{array}$

siRNA: Control $\overline{\mathrm{G \alpha}_{\mathrm{q} / 11}} \frac{\mathrm{Ga}_{\mathrm{s}}}{\mathrm{G}}$

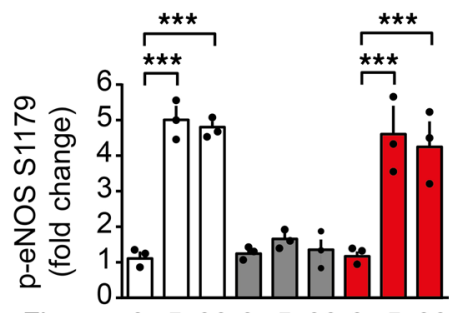

Flow: $\quad 0 \quad 5 \quad 300 \begin{array}{lllll}0 & 50 & 0 & 5 & 30\end{array}$

siRNA: Control $\overline{G \alpha_{q / 11}} \frac{0}{G \alpha_{s}}$

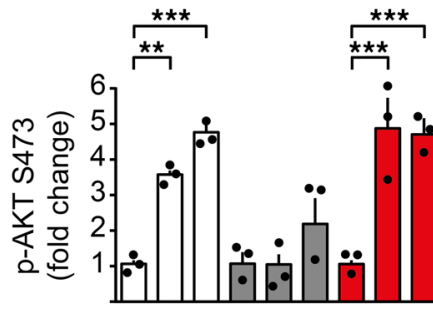

Flow: $0 \quad 5300053005530$

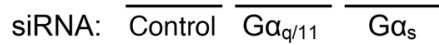

\section{B}
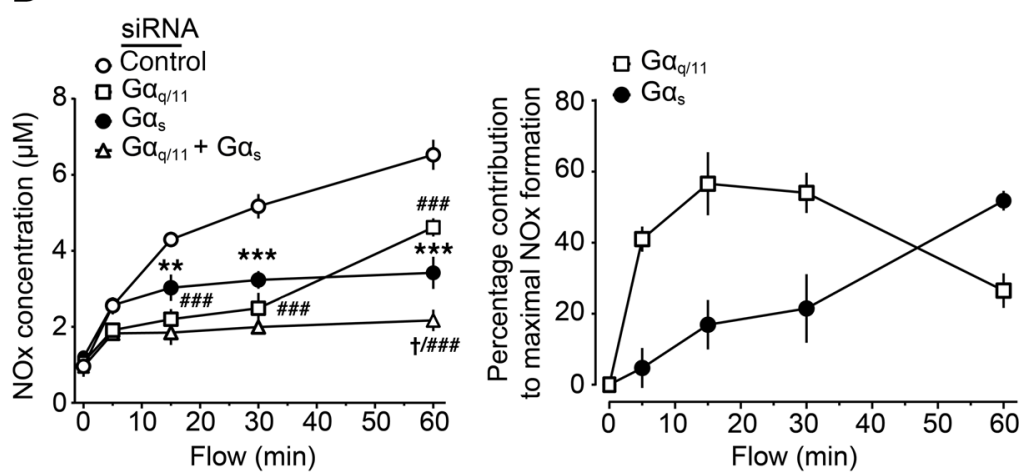

C

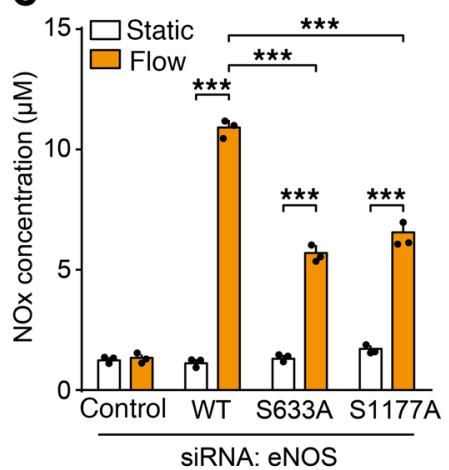

Figure 2. $G_{s}$ and $G_{q} / G_{11}$ additively regulate endothelial eNOS activity and NO formation. BAECs were transfected with control siRNA (control) or siRNA directed against $G \alpha_{s}$ or $G \alpha_{q}$ and $G \alpha_{11}$ as indicated. (A and B) Cells were exposed to $15 \mathrm{dyn} / \mathrm{cm}^{2}$ for the indicated time periods, and phosphorylation of eNOS and AKT was determined by immunoblotting (A). Bar diagrams show the densitometric evaluation $(n=3)$. (B) Nitrate and nitrite concentration in the cell culture medium $(n=9$, control; $n=6, G \alpha_{q} / G \alpha_{11} ; n=7, G \alpha_{s} ; n=5, G \alpha_{q} / G \alpha_{11}+G \alpha_{s}$ ) (left), and percentage contribution of $\mathrm{G}_{\mathrm{s}}$ and $G_{a} / G_{11}$ to the maximal nitrate and nitrite formation (right). (c) HAECs were transfected with siRNA directed against eNOS, and eNOS WT or the eNOS phospho-site mutants S1177A and S633A were expressed by lentiviral transduction. Thereafter, the effect of flow ( $15 \mathrm{dyn} / \mathrm{cm}^{2}$ for 30 minutes) on nitrate/nitrite concentration in the cell culture medium was determined $(n=3)$. Data represent the mean $\pm \mathrm{SEM} ;{ }^{*}, P \leq 0.05,{ }^{* *} P \leq 0.01,{ }^{* * *},{ }^{*} \# \#, \neq \neq \neq \leq 0.001,2$-way ANOVA with Bonferroni's post hoc test; ${ }^{*}$ and ${ }^{*}$, compared with control; ${ }^{\dagger}$, compared with siRNA against $\mathrm{G} \alpha_{\mathrm{s}} ;{ }^{\ddagger}$ compared with siRNA against $G \alpha_{q} / G \alpha_{11}$.

induction of endothelial $G \alpha_{s}$ deficiency on the telemetrically recorded blood pressure in conscious mice. Treatment of WT mice with tamoxifen resulted in a transient increase in arterial blood pressure. However, induction of endothelial $G \alpha_{s}$ deficiency led to a sustained increase of arterial blood pressure by 15-20 mmHg (Figure 3D). In parallel to the increase in arterial blood pressure after induction of endothelium-specific $G \alpha_{s}$ deficiency, we observed a reduced phosphorylation of murine aortic and mesenteric artery eNOS at serine 632, corresponding to serine 633 and serine 635 in human and bovine eNOS, respectively (Figure 3, E and F). Phosphorylation of murine eNOS at serine 1176, corresponding to serine 1177 and serine 1179 in human and bovine eNOS, respectively, was not affected by endothelial loss of $\mathrm{G}_{\mathrm{s}}$ (Figure 3, E and F). The reduced phosphorylation of eNOS at serine 632 in mice with induced endothelial $\mathrm{G} \alpha_{\mathrm{s}}$ deficiency was accompanied by a decrease in plasma nitrite/nitrate levels (Figure $3 \mathrm{G}$ and Supplemental Figure 1I). These data indicate that the acute loss of $\mathrm{G}_{\mathrm{s}}$-mediated signaling in the endothelium results in reduced eNOS activity, leading to increased vascular tone and blood pressure.

The adrenomedullin receptor CALCRL mediates endothelial fluid shear stress response in vitro. Since a role of $\mathrm{G}_{\mathrm{s}}$ in flow-induced endothelial responses strongly suggests the involvement of a GPCR operating upstream of $G_{s}$, we systematically analyzed the expression of GPCRs in BAECs and HUVECs (Supplemental Figure 2). Of the 91 GPCRs found to be expressed in both BAECs and HUVECs, we selected 45 receptors, which either were known to be $\mathrm{G}_{\mathrm{s}}$-coupled or were orphan receptors, and performed a siRNAmediated knockdown of these receptors (Figure 4A). While sup- 
A

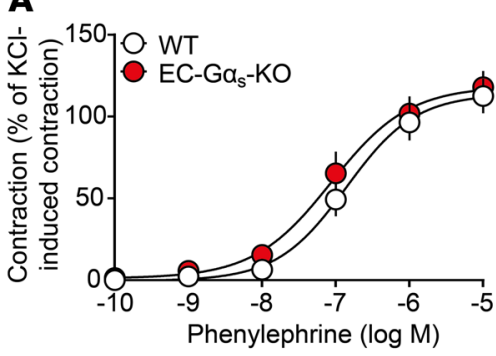

B

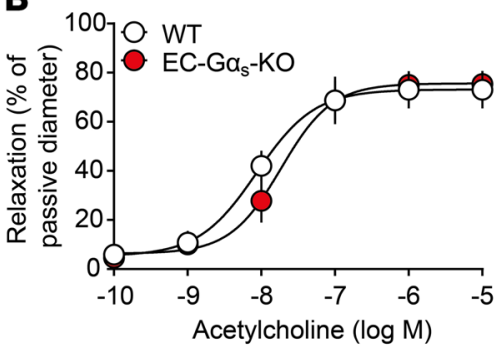

C

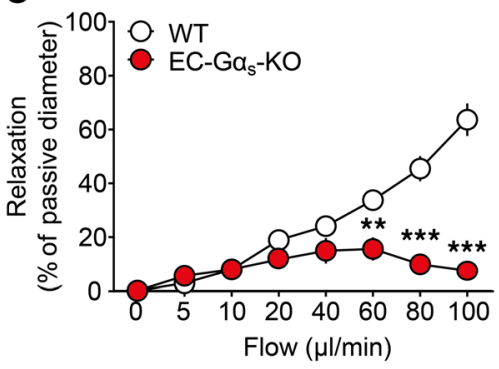

D

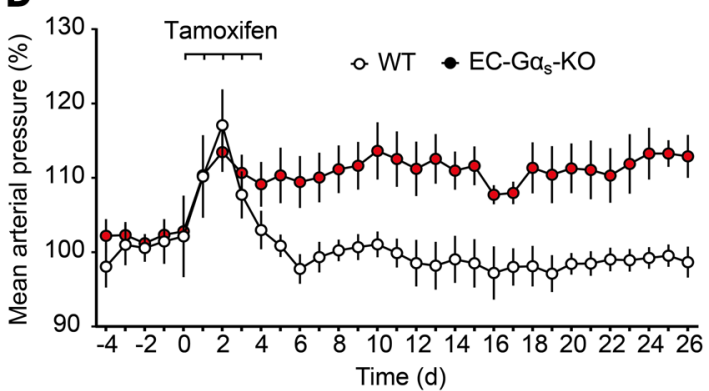

$\square$ Before induction $\square$ After induction
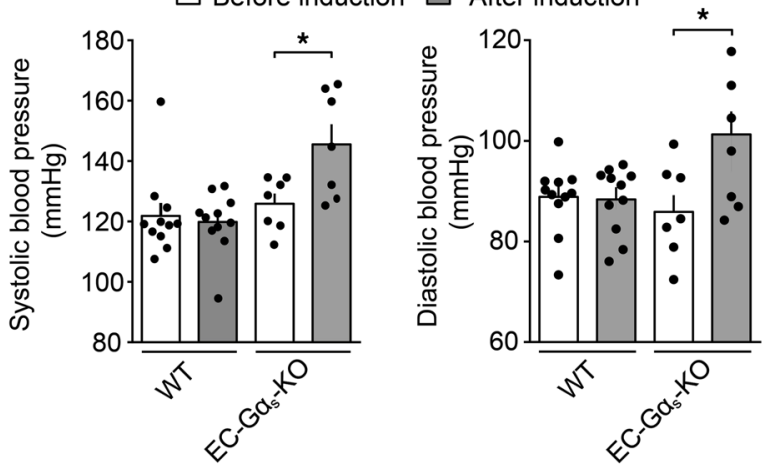

E

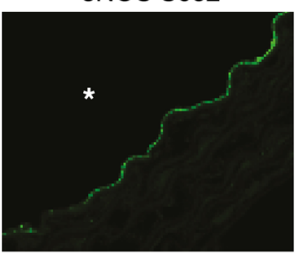

eNOS S1176

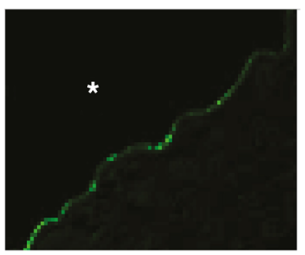

eNOS total

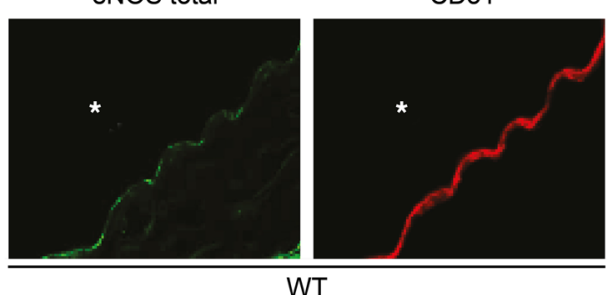

F

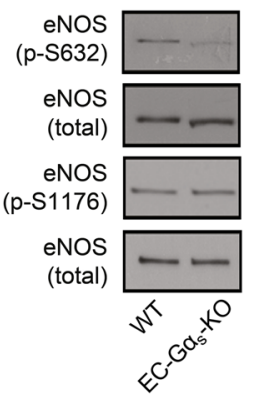

CD31

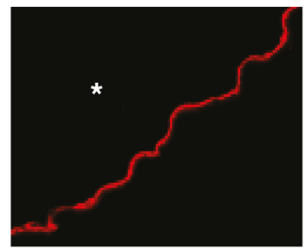

CD31

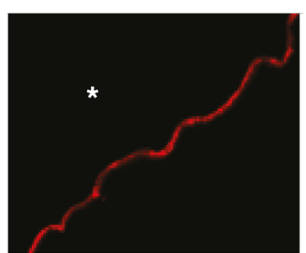

CD31

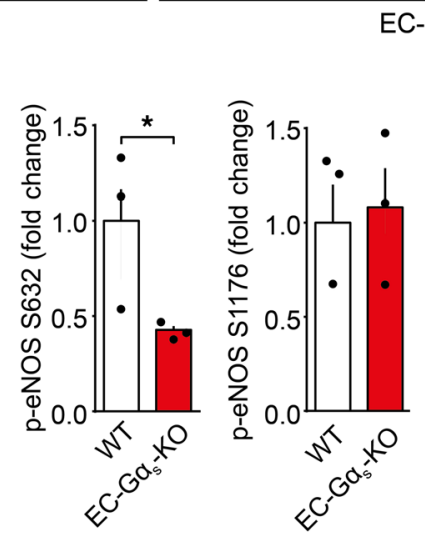

eNOS S632

eNOS S1176

eNOS total
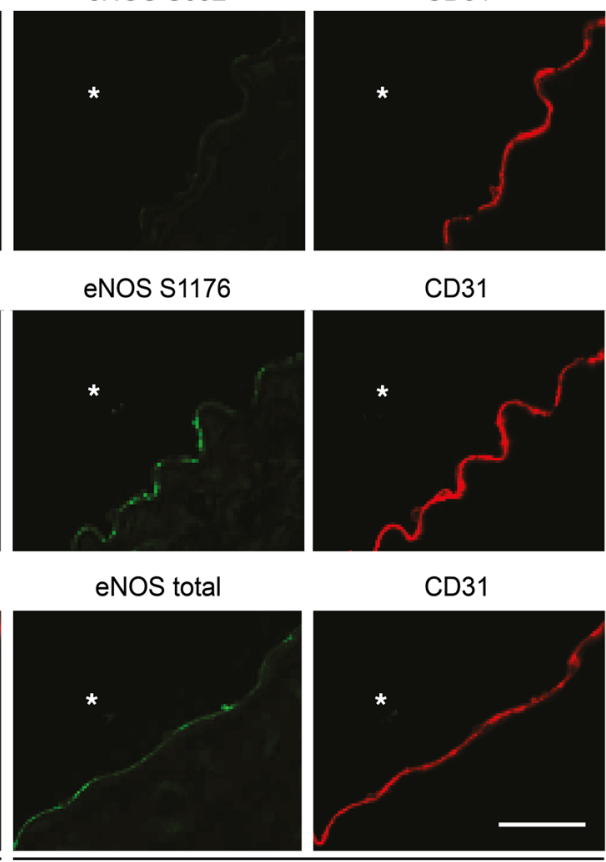

$\mathrm{EC}-\mathrm{Ga}_{\mathrm{s}}-\mathrm{KO}$

G
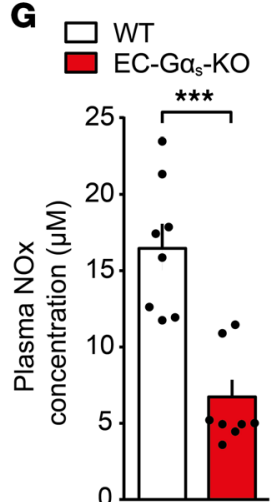

Figure 3. Endothelial $\mathbf{G} \alpha_{s}$ is required for flow-induced vasodilation and blood pressure control. (A and $\mathbf{B}$ ) Aorta segments from WT or induced EC- $\mathrm{C} \alpha_{\mathrm{s}}-\mathrm{KO}$ mice were incubated with increasing concentrations of phenylephrine $(\mathbf{A})$ or were precontracted with $10 \mu \mathrm{M}$ phenylephrine and exposed to acetylcholine (B), and vascular tension was determined $(n=5)$. (C) Mesenteric arteries isolated from tamoxifen-treated WT or EC- $\mathrm{G} \alpha_{\mathrm{s}}-\mathrm{KO}$ mice were precontracted with $100 \mathrm{nM}$ U46619 and exposed to stepwise increases in perfusion flow. Shown is the flow-induced vasodilation as a percentage of the passive vessel diameter ( $n=11, \mathrm{WT} ; n=7$, $\left.E C-G \alpha_{s}-K O\right)$. (D) Mean arterial blood pressure measured in conscious, freely moving WT ( $n=11)$ and EC- $\mathrm{C} \alpha_{\mathrm{s}}-\mathrm{KO}$ mice $(n=7)$ before, during, and after tamoxifen treatment. Bar diagrams show systolic and diastolic arterial blood pressure during 4 days before tamoxifen treatment and during the second week after induction. (E) Immuno-confocal microscopy images of aortae isolated from WT or EC-G $\alpha_{5}-$ KO mice stained with antibodies directed against eNOS, phosphorylated eNOS (S632 or \$1176, green), and the endothelial marker CD31 (red). Shown is 1 of 2 independent experiments. Scale bar: $25 \mu \mathrm{m}$. (F) Phosphorylation of eNOS at serine 632 and 1176 in lysates from mesenteric arteries prepared from tamoxifen-treated WT and EC-C $\alpha_{s}-K O$ mice. Graphs show the densitometric evaluation $(n=3)$. (G) Plasma nitrate and nitrite (NOx) levels in WT $(n=8)$ and EC-G $\alpha_{s}-$ KO mice $(n=8)$ 10 days after induction. Data represent the mean $\pm \mathrm{SEM} ;{ }^{*} P \leq 0.05,{ }^{*} P \leq 0.01$, ${ }^{* * *} P \leq 0.001,2$-way ANOVA with Bonferroni's post hoc test (A-C), paired 2-tailed Student's $t$ test (D), or unpaired 2-tailed Student's $t$ test (F and $\mathbf{G}$ ). 

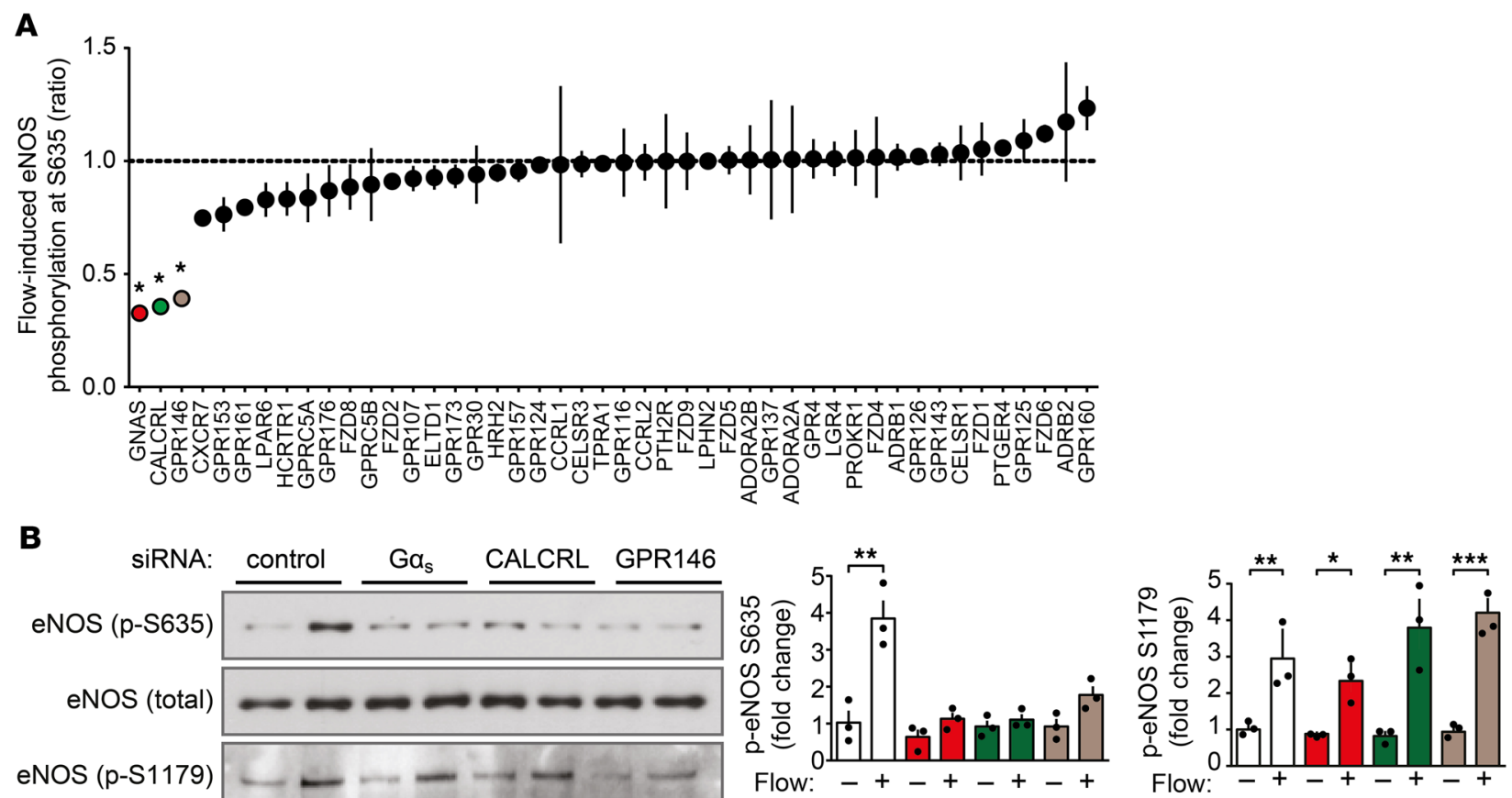

eNOS (p-S1179)

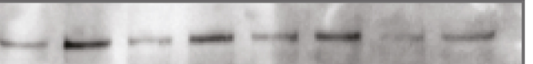

Flow:

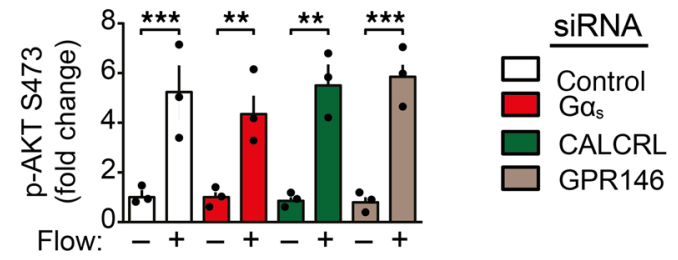

Flow: $-+-+\infty+-+$
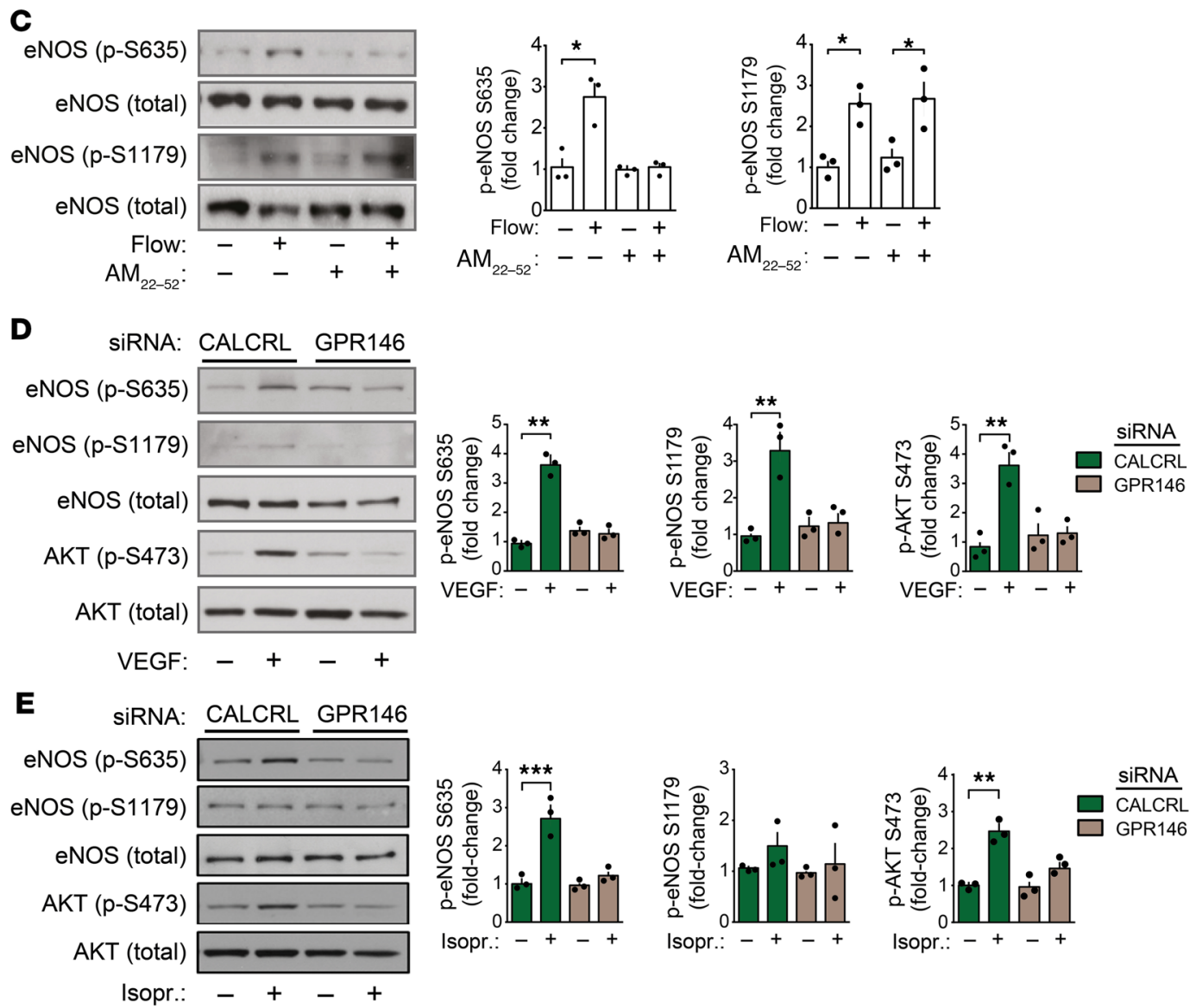
Figure 4. The adrenomedullin receptor CALCRL mediates endothelial fluid shear stress response in vitro. (A) BAECs were transfected with siRNAs against $\mathrm{G} \alpha_{\mathrm{s}}$ (GNAS) and the indicated GPCRs and were then exposed to flow $\left(15 \mathrm{dyn} / \mathrm{cm}^{2}\right)$ for 30 minutes. Shown is the ratio of flow-induced eNOS S635 phosphorylation in cells transfected with a control siRNA and with a siRNA against a GPCR. The plot shows the ranked average ratios of 3 independent experiments. (B-E) BAECs were transfected with control siRNA, siRNA directed against $G \alpha_{s}$, or an alternative siRNA against CALCRL and GPR146 (B, D, and $\mathbf{E}$ ) or were pretreated with the adrenomedullin receptor antagonist $\mathrm{AM}_{22-52}(1 \mu \mathrm{M})(\mathbf{C})$ and were exposed to flow $\left(15 \mathrm{dyn} / \mathrm{cm}^{2}\right)$ for 30 minutes (B and C) or to VEGF ( $50 \mathrm{ng} / \mathrm{ml}, 10$ minutes) (D) or to isoproterenol (Isopr., $10 \mu \mathrm{M}$, 10 minutes) (E). Total and phosphorylated eNOS and AKT were determined by immunoblotting. Graphs show the densitometric evaluation of blots $(n=3)$. Data represent the mean $\pm \mathrm{SEM}$; ${ }^{*} P \leq 0.05,{ }^{* *} P \leq 0.01$, ${ }^{* *} P \leq 0.001$, 1-way ANOVA with Dunnett's post hoc test (A), 2-way ANOVA with Bonferroni's post hoc test (B), or 1-way ANOVA with Tukey's post hoc test (C-E).

pression of the expression of the majority of the candidate receptors had no effect on flow-induced eNOS phosphorylation on serine 635 in BAECs, knockdown of the calcitonin receptor-like receptor (CALCRL) as well as of the orphan receptor GPR146 inhibited fluid shear stress-induced serine 635 phosphorylation to a similar degree to knockdown of $\mathrm{G} \alpha_{\mathrm{s}}$ (Figure $4 \mathrm{~A}$ ). An alternative siRNA directed against CALCRL and GPR146 as well as treatment with the CALCRL antagonist peptide $\mathrm{AM}_{22-52}$ blocked flow-induced eNOS phosphorylation at serine 635 (Figure 4, B and C, and Supplemental Figure 3A). However, knockdown of GPR146 also blocked $\mathrm{G}_{\mathrm{s}}$-independent phosphorylation of eNOS and AKT by VEGF (Figure 4D) as well as eNOS phosphorylation induced via $\beta$-adrenoceptors activated by isoproterenol (Figure 4E), whereas knockdown of CALCRL was without effect (Figure 4, D and E). Thus, we conclude that loss of flow-induced eNOS phosphorylation after knockdown of the orphan receptor GPR146 is due to unspecific or indirect effects. We therefore focused on CALCRL as a potential receptor mediating flowinduced $\mathrm{G}_{\mathrm{s}}$-dependent endothelial responses.

CALCRL is a GPCR that, together with receptor activitymodifying protein 1 (RAMP1), functions as a receptor for calcitonin gene-related peptide (CGRP), while RAMP2 and RAMP3 enable CALCRL to function as a receptor for adrenomedullin as well as adrenomedullin-2 (33). We therefore tested the effect of CGRP, adrenomedullin, and adrenomedullin-2 on phosphorylation of eNOS in endothelial cells and found that only adrenomedullin and adrenomedullin-2, but not CGRP, induced phosphorylation of serine 635 in BAECs (Figure 5A), an effect that was similar to flow-induced eNOS phosphorylation (Supplemental Figure 3B) and that was no longer observed after knockdown of $\mathrm{G} \alpha_{\mathrm{s}}$ and CALCRL expression (Figure 5, B and C). However, no phosphorylation of serine 1179 was observed (Figure 5, B and C), and in HAECs with suppressed eNOS expression, only the eNOS phospho-site mutant S1177A but not the S633A mutant was able to rescue adrenomedullin-induced NO formation in comparison with cells in which WT eNOS was re-expressed (Figure 5D and Supplemental Figure 1F). Of the 3 potential ligands, only adrenomedullin was expressed in endothelial cells (refs. 34, 35, and Figure $5 \mathrm{E}$ ). Knockdown of adrenomedullin blocked flow-induced eNOS phosphorylation at serine 635 but not eNOS phosphorylation at serine 1179 (Figure 5F and Supplemental Figure 3C). Similarly, knockdown of adrenomedullin and CALCRL strongly inhibited flow-induced cAMP formation (Figure 5G) as well as flow-induced NO formation (Figure $5 \mathrm{H}$ and Supplemental Figure 1E). Similar results were obtained when HAECs were used (Supplemental Figure 3, D and E). Thus, both, adrenomedullin and its receptor are required for flow-induced cAMP-mediated eNOS phosphorylation and activation.

Fluid shear stress induces adrenomedullin release from endothelial cells in a PIEZO1-dependent manner. When we exposed endothelial cells to flow, a 3 -fold increase in adrenomedullin release could be observed (Figure 6A). We further analyzed the mechanism through which fluid shear stress induces release of adrenomedullin from endothelial cells. Since the mechanosensitive cation channel PIEZO1 has been shown to play an important role in endothelial responses to fluid shear stress $(14,15)$, we incubated BAECs with siRNA against PIEZO1 and found that knockdown of PIEZO1 inhibited fluid shear stress-induced eNOS phosphorylation of serine 635 and of serine 1179 (Figure 6B and Supplemental Figure 4A). Furthermore, administration of the selective PIEZO1 agonist Yoda1 induced eNOS phosphorylation at serine 635 and serine 1179 (Figure 6C). However, endothelial cells lacking $\mathrm{G} \alpha_{\mathrm{s}}$ failed to respond with eNOS phosphorylation of serine 635 upon Yoda1 treatment, whereas Yoda1 was still able to induce serine 1179 phosphorylation (Figure 6C). Yoda1-induced eNOS phosphorylation at serine 635 was also blocked by the adrenomedullin receptor antagonist $\mathrm{AM}_{22-52}$ as well as after knockdown of CALCRL or adrenomedullin (Supplemental Figure 4, B and C). Yoda1-induced NO formation in HAECs was abrogated after knockdown of eNOS and partially rescued by re-expression of the eNOS phospho-site mutants S633A and S1177A compared with re-expression of WT eNOS (Figure 6D and Supplemental Figure 1F). The Yoda1 effects were not seen after knockdown of PIEZO1. These data indicate that PIEZO1 critically contributes not only to fluid shear stress-induced ATP release and subsequent $\mathrm{P}_{2} \mathrm{Y}_{2} /$ $\mathrm{G}_{\mathrm{q}} / \mathrm{G}_{11}$-dependent eNOS phosphorylation at serine 1179 (16) but is also required for fluid shear stress-induced adrenomedullin release and subsequent $\mathrm{G}_{\mathrm{s}}$-dependent regulation of eNOS phosphorylation at serine 635. Flow-induced release of adrenomedullin from endothelial cells occurs in a $\mathrm{Ca}^{2+}$-dependent manner, as it was blocked by pretreatment of cells with the $\mathrm{Ca}^{2+}$ chelator BAPTA-AM (Figure 6E) and could be mimicked by the $\mathrm{Ca}^{2+}$ ionophore A23187 (Supplemental Figure 4D). Also, the PIEZO1 activator Yoda1 was able to induce adrenomedullin release (Figure 6F). Both flow- and Yoda1-induced adrenomedullin releases were blocked after knockdown of PIEZO1 expression (Figure 6, A and F), but not after knockdown of $\mathrm{G} \alpha_{q} / \mathrm{G \alpha}_{11}$ (Supplemental Figure $4 \mathrm{E}$ ). To further analyze the role of PIEZO1 in fluid shear stress-induced adrenomedullin release, we determined plasma levels of adrenomedullin in WT mice and in mice with induced endotheliumspecific PIEZO1 deficiency (16). Mice lacking PIEZO1 in endothelial cells showed an almost 50\% reduction in plasma adrenomedullin levels (Figure 6G). This strongly indicates that PIEZO1 is involved in flow-induced adrenomedullin release.

Adrenomedullin and its receptor mediate flow-induced vasodilation and control basal blood pressure. We then generated inducible endothelium-specific CALCRL-deficient mice (Tek-CreER ${ }^{T 2}$;$\mathrm{Calcrl}^{\mathrm{l} / \mathrm{fl}}$, herein referred to EC-Calcrl-KO mice; Supplemental Figure 5, A and B) as well as inducible endothelium-specific adre- 
A

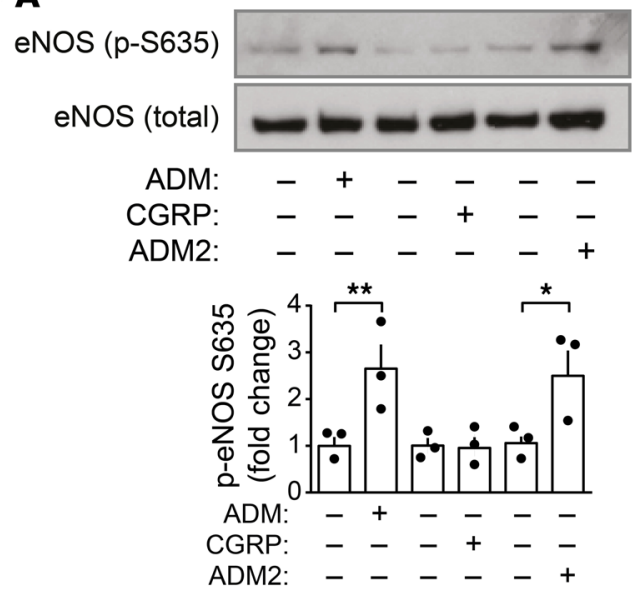

B

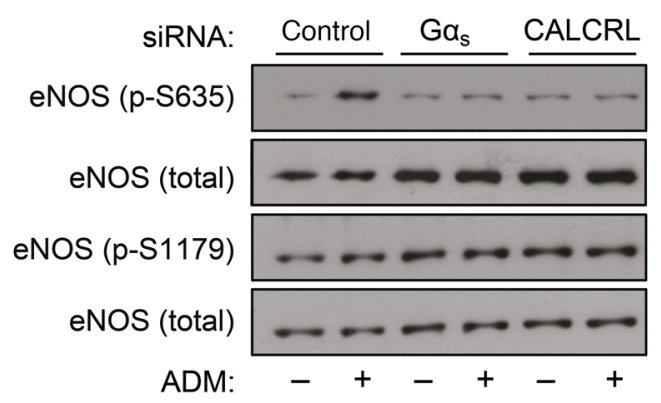

siRNA: $\square$ Control $\square \mathrm{Ga}_{\mathrm{s}} \square$ CALCRL

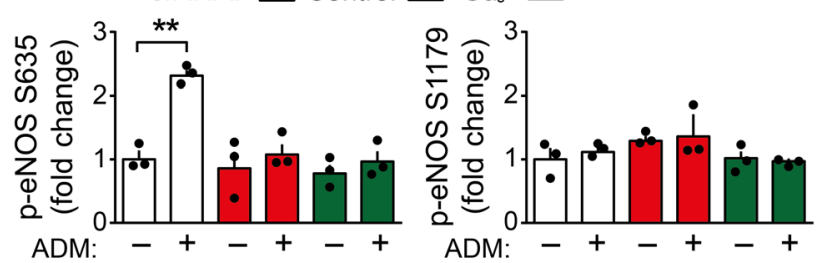

C
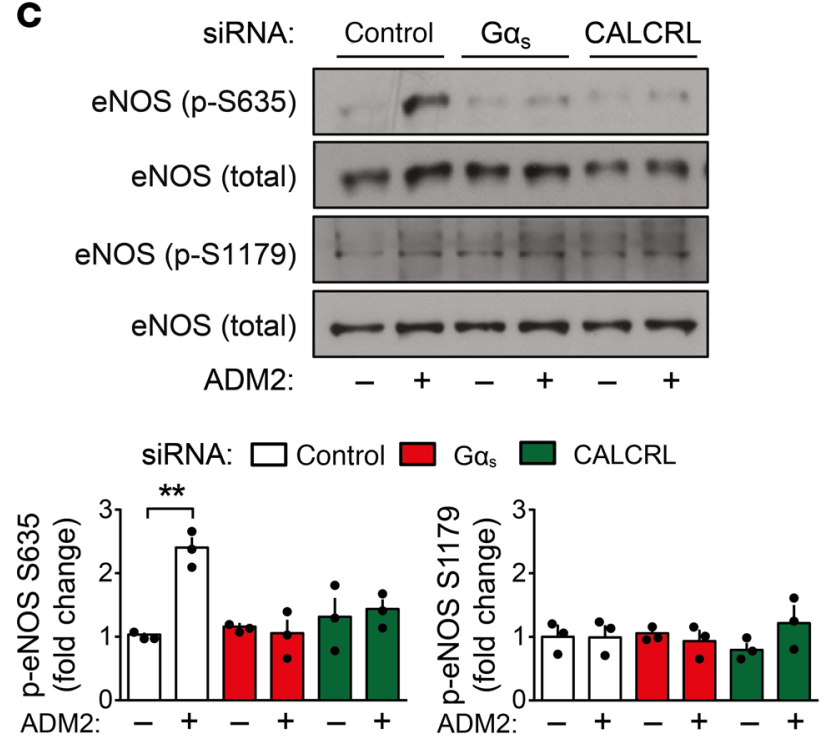

D

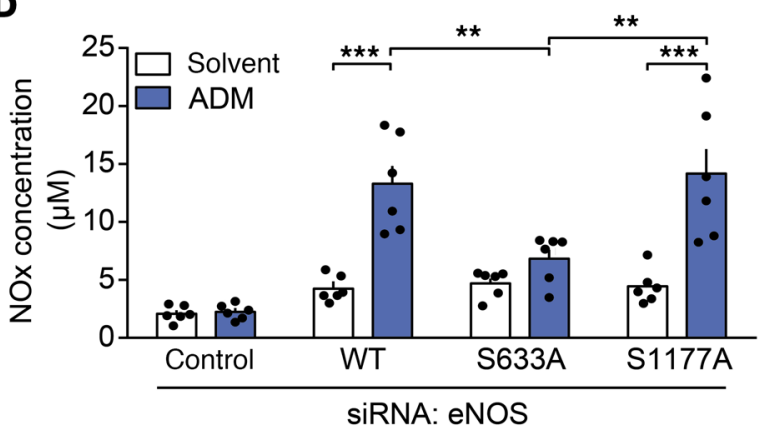

E

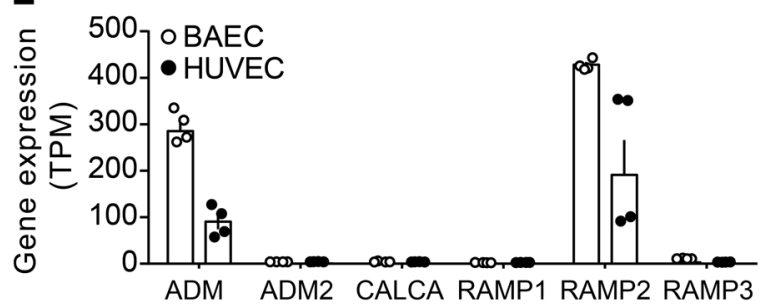

$\mathbf{F}$

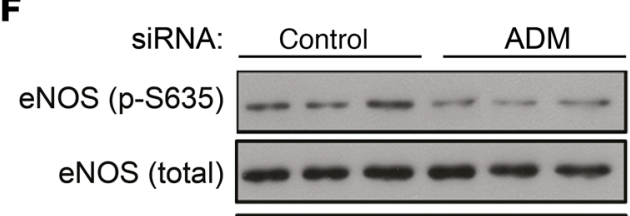

eNOS (p-S1179)

eNOS (total)

Flow: $\begin{array}{llllll}0 & 5 & 30 & 0 & 5 & 30\end{array}$ siRNA: $\square$ Control $\square$ ADM

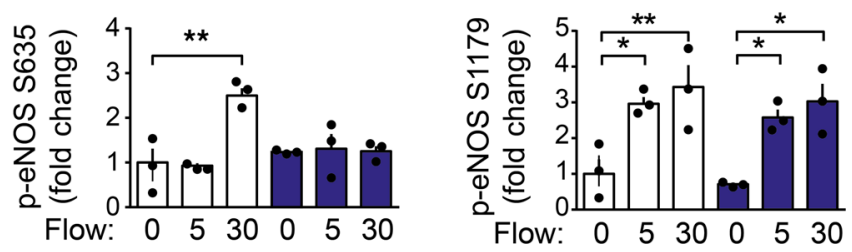

G
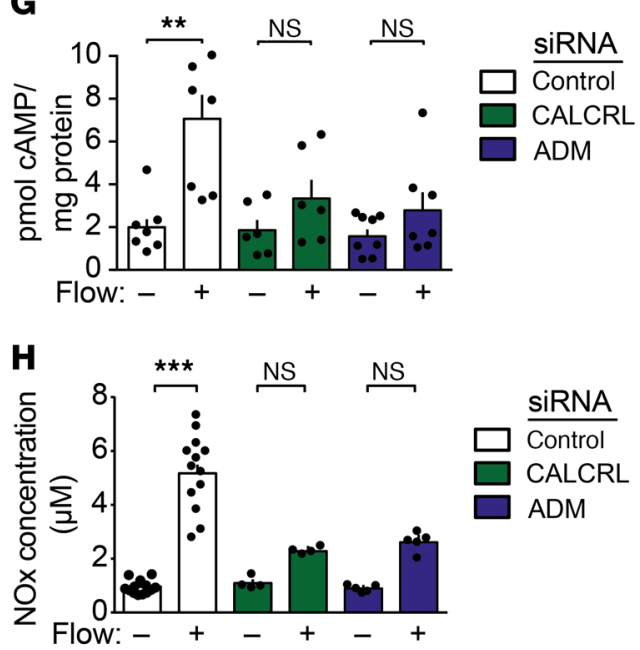
Figure 5. Role of adrenomedullin in flow-induced eNOS regulation.

(A) BAECs were treated with adrenomedullin (ADM, $10 \mathrm{nM}, 5$ minutes), calcitonin gene-related peptide (CCRP; $10 \mathrm{nM}, 10$ minutes), or adrenomedullin-2 (ADM2, $1 \mathrm{nM}, 3$ minutes), and phosphorylation of eNOS S635 was determined by immunoblotting. Bar diagram shows the densitometric evaluation $(n=3)$. (B-D and $\mathbf{F}-\mathbf{H})$ BAECs (B, C, and $\mathbf{F}-\mathbf{H})$ or HAECs (D) were transfected with scrambled (control) siRNA or siRNA directed against $G \alpha_{s^{\prime}}$ CALCRL, eNOS, or ADM as indicated. In $\mathbf{D}$, eNOS WT or the eNOS phospho-site mutants S1177A and S633A were expressed by lentiviral transduction. Cells were treated with adrenomedullin (ADM, $10 \mathrm{nM}, 5 \mathrm{~min}-$ utes [B] or 30 minutes [D]) or adrenomedullin-2 (ADM2, $1 \mathrm{nM}, 3$ minutes, C) or were exposed to $15 \mathrm{dyn} / \mathrm{cm}^{2}$ for 30 minutes or for the indicated time periods (F-H). Phosphorylation of eNOS at serine 635 and serine 1179 was determined by immunoblotting (B, C, and $\mathbf{F}$ ). Intracellular cAMP concentration ( $n=7$, control; $n=6$, CALCRL; $n=8, \mathrm{ADM}$ ) (G) or nitrate and nitrite concentration in the cell culture medium ( $n=6[\mathrm{D}] ; n=13$, control; $n=4$, CALCRL; $n=5$, ADM $[\mathbf{H}]$ ) was determined. Bar diagrams in $\mathbf{B}, \mathbf{C}$, and $\mathbf{F}$ show densitometric evaluation of immunoblots $(n=3)$. (E) Expression of ADM, CGRP (CALCA), ADM2, and RAMP1-3 RNA in BAECs and HUVECs $(n=4)$. Data represent the mean $\pm \mathrm{SEM}$; ${ }^{*} P \leq 0.05$, ${ }^{* *} P \leq 0.01$, ${ }^{* *} P \leq 0.00$, 2-way ANOVA with Bonferroni's post hoc test (A-D, $\mathbf{G}$, and $\mathbf{H})$ or 1-way ANOVA with Tukey's post hoc test (F).

nomedullin-deficient mice $\left(T e k-C r e E R^{T 2} ; A d m^{f / f l}\right.$, herein referred to as EC-Adm-KO mice) to investigate the physiological role of adrenomedullin and CALCRL in fluid shear stress-induced eNOS activation. Aortic segments from EC-Calcrl-KO and EC-Adm-KO mice showed normal contraction in response to phenylephrine and, after precontraction, responded normally to the vasodilator acetylcholine (Supplemental Figure 6). However, flow-induced vasodilation of mesenteric arteries was strongly reduced (Figure 7, A and B). When endothelium-specific CALCRL and adrenomedullin deficiency was induced in vivo, the phenotype of induced endothelium-specific $G \alpha_{s}$ deficiency was recapitulated, and animals showed an increase in arterial blood pressure by about 10-20 $\mathrm{mmHg}$ (Figure 7, C and D) accompanied by a reduced phosphorylation of murine aortic eNOS at serine 632 but not at serine 1176 (Figure 7E) and a reduction in plasma nitrite/nitrate levels (Figure $7 \mathrm{~F}$ and Supplemental Figure 1I). While free plasma adrenomedullin levels were reduced in EC-Adm-KO mice, EC-Calcrl-KO animals showed increased levels (Figure $7 G$ ). These data strongly indicate that adrenomedullin and CALCRL are acting upstream of endothelial $\mathrm{G}_{\mathrm{s}}$ in fluid shear stress-dependent regulation of $\mathrm{NO}$ formation, vascular tone, and blood pressure.

\section{Discussion}

Fluid shear stress exerted by the flowing blood is an important stimulus for the endothelium to release vasoactive factors, such as NO, and to thereby control vascular functions including vascular tone and blood pressure. Previous work has identified several mechanotransduction processes able to regulate eNOS activity in response to fluid shear stress $(3,18)$. These studies have mainly focused on $\mathrm{Ca}^{2+}$-mediated pathways and eNOS regulation involving AKT $(8,9)$. However, eNOS can also be phosphorylated and regulated by PKA $(3,18,36)$, but the physiological role of this regulation and its upstream control mechanisms have remained unknown. Here we show that the endothelial adrenomedullin receptor CALCRL and $G_{s}$ mediate fluid shear stress-induced increases in cAMP levels, resulting in PKA-dependent eNOS phos- phorylation and activation. We also demonstrate that fluid shear stress induces adrenomedullin release from endothelial cells via activation of the mechanosensitive cation channel PIEZO1, and the released adrenomedullin then activates its receptor on endothelial cells. The fact that mice lacking PIEZO1 in endothelial cells have reduced adrenomedullin plasma levels and that acute loss of endothelial adrenomedullin-, CALCRL-, and Gs-mediated signaling leads to strongly reduced eNOS activity and to hypertension in vivo indicates that the adrenomedullin/ $\mathrm{G}_{\mathrm{s}}$-mediated signaling pathway in endothelial cells is constantly activated to mediate fluid shear stress-induced endothelial NO formation and vasodilation (Figure 8).

Endothelial NO formation by eNOS is regulated by different pathways that alter phosphorylation of the enzyme $(3,4,18,37)$. It has previously been shown that fluid shear stress induces phosphorylation of serine 635 of bovine eNOS through PKA (24), and that phosphorylation of serine 635 by PKA enhances eNOS activity (19-21, 24). Our data show that flow-induced PKA-dependent eNOS activation is mediated by the $\mathrm{G}_{\mathrm{s}}$-coupled adrenomedullin receptor, which promotes formation of the PKA activator cAMP. Flow-induced phosphorylation of human eNOS at serine 1177, which also increases eNOS activity, is in turn controlled by the $\mathrm{G}_{\mathrm{q}} / \mathrm{G}_{11}$-coupled P2Y receptor activated by ATP released in response to flow (17). This indicates that flow-induced phosphorylation of human eNOS at serine 633 and 1177 through $\mathrm{G}_{\mathrm{s}}$ - and $\mathrm{G}_{\mathrm{q}} / \mathrm{G}_{11}$-mediated signaling, respectively, occurs independently of each other. This is in contrast to data that showed that the PKA inhibitor H89 can block flow-induced phosphorylation of bovine eNOS not only at serine 635 but also at serine 1179 (38). This finding may be explained by the rather unspecific activity of H89, which also blocks the activity of several other protein kinases, including AKT (39), which mediates phosphorylation of serine $1177(8,9)$. Consistent with a specific role of PKA in phosphorylating eNOS at serine 633/635, we found that several other stimuli resulting in an elevation of cAMP, such as forskolin and isoproterenol, induced only eNOS phosphorylation at serine 633/635 but not at serine 1177. Also, the specific PKA inhibitor peptide PKI inhibited only flow-induced phosphorylation of serine 633/635. The fact that under in vivo conditions eNOS is phosphorylated at both sites and that induced endothelial loss of $\mathrm{G}_{\mathrm{s}}$ in mice is accompanied by strongly reduced eNOS phosphorylation at serine 632 but not at serine 1176 whereas phosphorylation at serine 1176 is affected by induced endothelial loss of $G \alpha_{q} / G \alpha_{11}$ (17) suggests that these 2 pathways act in an additive or synergistic manner (Figure 8). Phosphomimetic mutations at serine 635 and 1179 of bovine eNOS have indeed been shown to synergistically activate the enzyme (22). This is consistent with our in vitro data showing that knockdown of both $\mathrm{G} \alpha_{\mathrm{q}} / \mathrm{G \alpha}_{11}$ and $\mathrm{G} \alpha_{\mathrm{s}}$ strongly inhibited flow-induced NO formation, whereas knockdown of $\mathrm{G} \alpha_{\mathrm{q}} / \mathrm{G \alpha}_{11}$ or $\mathrm{Ga}_{s}$ alone had only a partial effect on flow-induced NO-formation.

Our initial observation that endothelial loss of CALCRL and $\mathrm{G} \alpha_{s}$ blocks flow-induced eNOS phosphorylation and inhibits NO formation raises the question of how fluid shear stress induces CALCRL activation. Some GPCRs, such as bradykinin B2, the

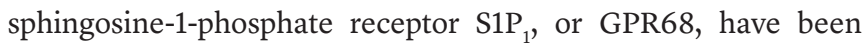
shown to be activated by flow in a ligand-independent manner $(40-42)$. The fact that the flow-induced $\mathrm{G}_{\mathrm{s}}$-mediated effect can 
A

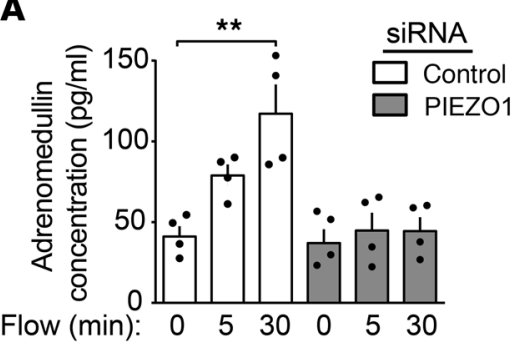

B

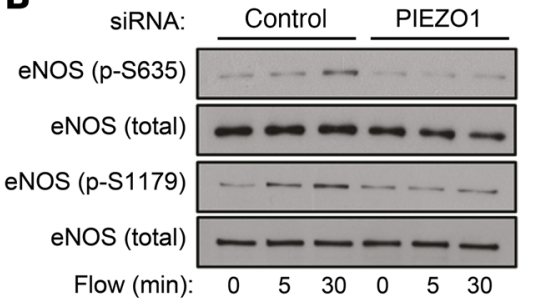

siRNA: $\square$ Control $\square$ PIEZO1

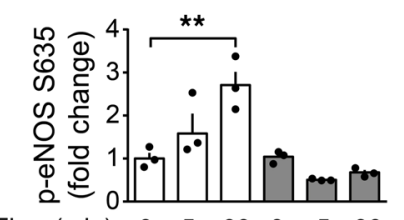

Flow (min): $0 \quad \begin{array}{llllll}0 & 5 & 30 & 0 & 5 & 30\end{array}$

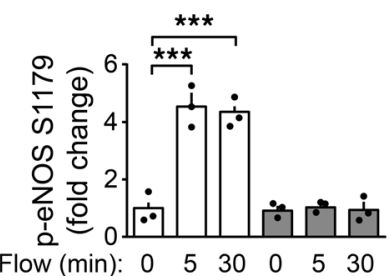

C
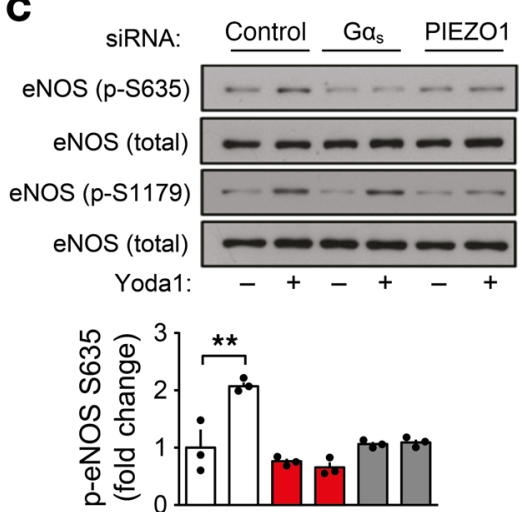

Yoda1: $-+\ldots+\ldots$

siRNA: Control $\mathrm{Ga}_{\mathrm{s}}$ PIEZO1

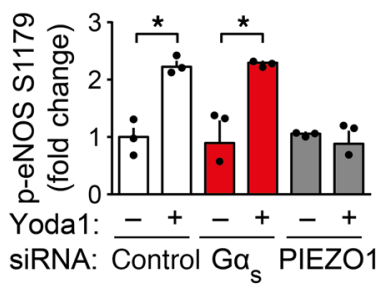

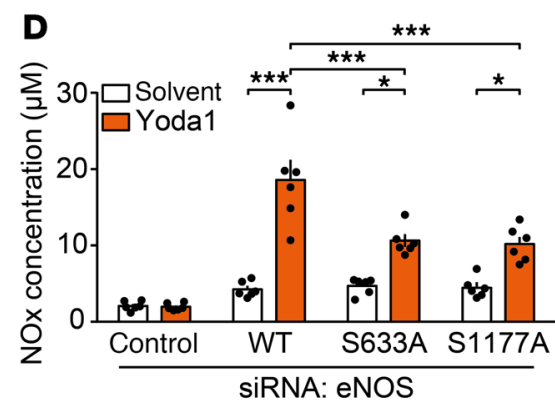

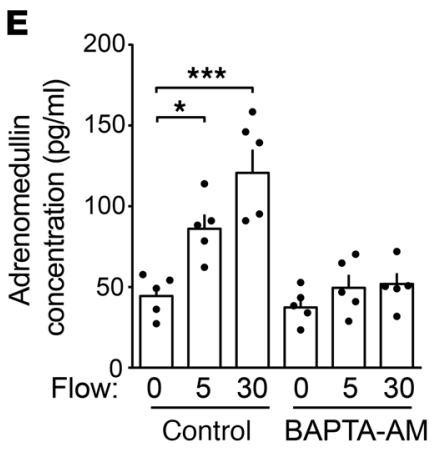

$\mathbf{F}$

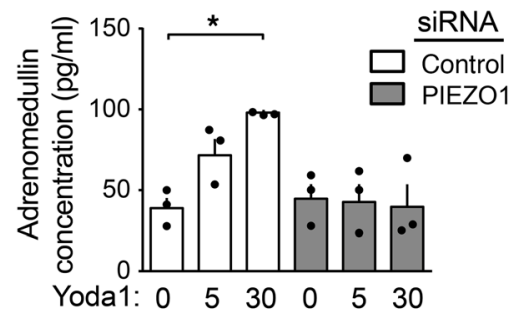

G

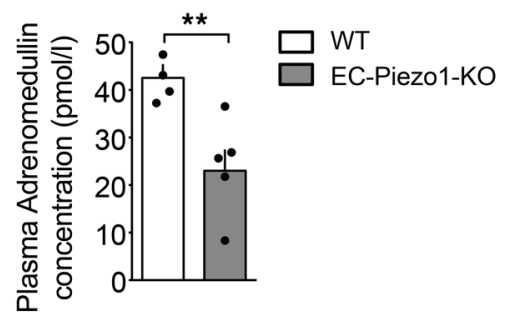

Figure 6. Mechanism of flow-induced adrenomedullin release from endothelial cells. (A-F) BAECs (A-C, E, and $\mathbf{F}$ ) or HAECs (D) were transfected with scrambled (control) siRNA or siRNA directed against $\mathrm{G} \alpha_{\mathrm{s}}$, PIEZO1, or eNOS as indicated (A-D and $\mathbf{F}$ ) or were pretreated in the absence or presence of BAPTA-AM $(100 \mu \mathrm{M})$ for 20 minutes (E). In D, eNOS WT or the eNOS phospho-site mutants S1177A and S633A were expressed by lentiviral transduction. Cells were then exposed to flow (15 $\left.\mathrm{dyn} / \mathrm{cm}^{2}\right)(\mathbf{A}, \mathbf{B}$, and $\mathbf{E})$ for the indicated time periods or were incubated with Yoda1 $(1 \mu \mathrm{M})$ for 30 minutes (C, D, and $\mathbf{F})$. Thereafter the adrenomedullin concentration in the cell culture medium was determined (A, E, and F), phosphorylation of eNOS serine 635 and serine 1179 was determined by immunoblotting (B and $\mathbf{C}$ ), or the nitrate and nitrite concentration in the cell culture medium was determined (D). Bar diagrams show adrenomedullin concentration ( $n=4$ in $\mathbf{A}, n=5$ in $\mathbf{E}$, and $n=3$ in $\mathbf{F}$ ) or the densitometric evaluation (B and C; $n=3)$ or the nitrate/nitrite concentration ( $n=6$ in D). (C) Plasma adrenomedullin concentration in WT $(n=4)$ and EC-Piezo1-KO mice $(n=5) 10$ days after induction. Data represent the mean $\pm \mathrm{SEM} ;{ }^{*} P \leq 0.05,{ }^{* *} P \leq 0.01$, ${ }^{* * *} P \leq 0.001$, 1-way ANOVA with Tukey's post hoc test (A, B, E, and F), 2-way ANOVA with Bonferroni's post hoc test (C and $\mathbf{D})$, or 2-tailed Student's $t$ test (C).

be blocked by the competitive adrenomedullin receptor antagonist $\mathrm{AM}_{22-52}$ suggests that flowinduced $\mathrm{G}_{\mathrm{s}}$-mediated signaling requires the presence of adrenomedullin. This is consistent with previous observations that increased fluid shear stress on endothelial cells induced expression and release of adrenomedullin (43-46). A central role of adrenomedullin in flow-induced eNOS activation via CALCRL and $G_{s}$ is also indicated by our findings that fluid shear stress induces the release of adrenomedullin from endothelial cells and that suppression of endothelial adrenomedullin expression in vitro and in vivo recapitulates the effect of endothelial loss of CALCRL and $\mathrm{G}_{\mathrm{s}}$.

The mechanism of flow-induced adrenomedullin release from endothelial cells remains to be further elucidated but involves the mechanosensitive cation channel PIEZO1, as knockdown of PIEZO1 blocked flow-induced adrenomedullin release. The critical role of PIEZO1 in flow-induced adrenomedullin release from endothelial cells is also indicated by our observation that plasma levels of adrenomedullin drop by about $50 \%$ after induction of endotheliumspecific PIEZO1 deficiency. We have recently shown that PIEZO1 is also required for flowinduced ATP release from endothelial cells, which acts through $\mathrm{G} \alpha_{q} / \mathrm{G \alpha}_{11}$-coupled P2Y $\mathrm{Y}_{2}$ receptors (16). However, flow-induced adrenomedullin release was not affected by knockdown of $\mathrm{G} \alpha_{\mathrm{q}} /$ $\mathrm{G}_{11}$ in endothelial cells. Thus, we conclude that flow-induced endothelial adrenomedullin release 
requires PIEZO1 activation and so far poorly understood downstream mechanisms to mediate flow-induced CALCRL/G $/ \mathrm{G}_{\mathrm{s}}$ activation. The fact that flow-induced adrenomedullin release from endothelial cells depended on the cation channel PIEZO1, could be mimicked by a $\mathrm{Ca}^{2+}$ ionophore, and was blocked by the intracellular $\mathrm{Ca}^{2+}$ chelator BAPTA-AM indicates the involvement of a $\mathrm{Ca}^{2+}-$ mediated release mechanism for adrenomedullin in endothelial cells. Such a mechanism has been described for the release of adrenomedullin from adrenal medullary cells (47). The exact mechanism of $\mathrm{Ca}^{2+}$-mediated adrenomedullin release from endothelial cells is not known. However, recent evidence indicates that the actin-binding protein cortactin, which translocates in response to fluid shear stress to the cortical actin layer of endothelial cells (48) and which promotes the secretion of exosomes and various proteins (49-52), is required for adrenomedullin release from endothelial cells (53).

Adrenomedullin is considered a vasoprotective factor, which, besides its vasodilatory activity, promotes vascular integrity and inhibits vascular permeability $(54,55)$. However, the mechanism of adrenomedullin release and action had remained unclear. Free plasma levels of adrenomedullin in humans are in the range of 3-6 pM (56-59), far too low to activate CALCRL and RAMP2/3, which require adrenomedullin concentrations of about $1 \mathrm{nM}$ for half-maximal activation $(33,60)$. Our data indicate that adrenomedullin release from the endothelium is controlled by the blood flow acting through PIEZO1 and that adrenomedullin acts in an autocrine or paracrine manner. A constitutive flow-dependent autocrine or paracrine mechanism is also indicated by our observation of strong defects in fluid shear stress-induced cellular effects in isolated endothelial cells with suppressed adrenomedullin and adrenomedullin receptor expression as well as in flow-induced vasodilation in vessels isolated from endothelium-specific CALCRL- and adrenomedullin-deficient mice. Flow-induced adrenomedullin-dependent cAMP production and eNOS activation may also be critically involved in other adrenomedullin functions that have been shown to require endothelial adrenomedullin release and functional endothelial adrenomedullin receptors, such as angiogenesis and maintenance of endothelial barrier function (61-64).

A fundamental function of the endothelial layer of blood vessels is their ability to sense fluid shear stress and to transform this information into intracellular signals. These mechanosensing and -signaling processes are critical for maintaining vascular integrity, affect multiple vascular functions such as vascular tone and morphogenesis, and have a strong effect on pathological vascular processes including hypertension and atherosclerosis. We have discovered a novel endothelial mechanosignaling pathway consisting of PIEZO1, the adrenomedullin receptor, and $\mathrm{G}_{\mathrm{s}}$-mediated signaling that links fluid shear stress to the regulation of eNOS activity and is required for physiological regulation of vascular tone and blood pressure. In the future, it will be interesting to explore the role of this mechanosignaling pathway in other vascular functions requiring the ability of endothelial cells to respond to fluid shear stress, and to investigate its role in corresponding human vascular diseases.

\section{Methods}

Reagents. Acetylcholine, sodium nitroprusside, isoprenaline (isoproterenol), phenylephrine, adrenomedullin, BAPTA-AM [1,2-bis(2-amino- phenoxy) ethane- $N, N, N^{\prime}, N^{\prime}$-tetraacetic acid tetrakis(acetoxymethyl ester)], A23187, and tamoxifen were purchased from Sigma-Aldrich. Recombinant human VEGF was purchased from PeproTech $\mathrm{GmbH}$ (catalog 100-20). Myristoylated PKA inhibitor 14-22 (PKI) was purchased from Merck Chemicals GmbH (catalog 476485). H89 was obtained from Biomol GmbH (catalog Cay10010556). Human $\mathrm{AM}_{22-52}$ was purchased from Bachem (catalog H-4144). U46619 was from Cayman Chemical (catalog 56985-40-1). Adrenomedullin-2 (intermedin) was obtained from Phoenix Europe $\mathrm{GmbH}$ (catalog 010-48); CGRP was from Tocris Bio (catalog 1161). Antibodies directed against phosphorylated eNOS (mouse S1176, human S1177, and bovine S1179; catalog 9571), phosphorylated AKT (S473; catalog 4060), AKT (catalog 9272), and GAPDH (catalog 2118) were obtained from Cell Signaling Technology. Antibody directed against phosphorylated eNOS (mouse S632, human S633, and bovine S635; catalog 07-562) was obtained from EMD Millipore or from BD Biosciences (catalog 612664). AntieNOS (catalog 610296) and anti-CD31 (PECAM-1) antibodies (cata$\log 557355)$ were purchased from BD Biosciences. Anti-G $\alpha_{\mathrm{s}}$ antibody (catalog sc-823) and anti-G $\alpha_{q} / G \alpha_{11}$ antibody (catalog sc-392) were purchased from Santa Cruz Biotechnology Inc.

Primary cells. HUVECs and HAECs (Lonza) were cultured in EGM-2 medium, and BAECs (Lonza) were cultured in EGM-2 MV medium (Lonza). Cells were used for a maximal number of 6 passages.

siRNA-mediated knockdown. Cells at $70 \%$ confluence were transfected with siRNA using Opti-MEM and Lipofectamine RNAiMAX (Invitrogen) as described previously (17). siRNAs used for screening were pools of 3 siRNAs directed against 45 GPCRs (either $\mathrm{G}_{\mathrm{s}}$-coupled or orphan) that show high expression in BAECs and HUVECs and were obtained from Sigma-Aldrich. The targeted sequences are shown in Supplemental Table 1. Other siRNAs used were against the following bovine sequences: 5-GCATCCGAGTCAAGCGCTA-3, 5-CCGCCAGAGTTTGAACAAC-3, 5-GGACATGCACGGTGCAGAA-3 (ADM); 5-GCCCTTCCTGCAGTCTGGA-3, 5-CCGCCTGTGGCAGCTCGTT-3, 5-CGGTCACCCTCGGTTGCAT-3 (ADM2); 5-GAATCCAATCTGTACATGA-3, 5-GACTATAATTGGACATGTA-3, 5-GATCAGTTCTGATACGCAA-3 (CALCRL); 5-CCATGATGGCGTGTTGCCTGA-3, 5-CACGTTCATCAAGCAGATGCG-3, 5-CGACGCCCGGCGCGAGCTCAA-3， 5-GACCTGGAGAACATCATCTTC-3 (GNA11); 5-CACCAAGCTGGTGTATCAGAA-3， 5-AACAATTTGCATAATACTAAT-3，5-AAAGATCTTCTAGAGGAGAAA-3，5-CTGCCTGAGCGAGGAGGCCAA-3 (GNAQ); 5-CTGATTGACTGCGCCCAGT-3 (GNAS); 5-GCGCTAGTGACGCTGTACT-3, 5-CACTCTATGCGCTCGCGCT-3， 5-CCTACATGTCCAGCGTCTA-3 (GPR146); 5-GTGTTTGGTCTCAAGGACT-3， 5-GTGTCTACTTCCTGCTCTT-3 (PIEZO1).

Shear stress assays. For biochemical experiments to determine protein phosphorylation by immunoblotting, cells were exposed to flow using a parallel-plate flow chamber ( $\mu$-Slide I 0.4 Luer, ibidi). The flow chamber was perfused with a constant flow rate using a computercontrolled air pressure pump (ibidi). The constant wall shear stress ( $\tau$ ) is given by $\tau=\eta \times 131.6 \varphi$ ( $\eta$, viscosity of the medium; $\varphi$, flow rate) (according to the manufacturer's instructions).

For experiments to determine cAMP, nitrate/nitrite, and adrenomedullin levels, the BioTech-Flow System cone-plate viscometer (MOS Technologies, Germany) was used to expose cells to fluid shear stress as described previously (65). The cone of this system has an angle of $2.5^{\circ}$ and rotates on top of a $33-\mathrm{cm}^{2}$ cell culture dish contain- 
A

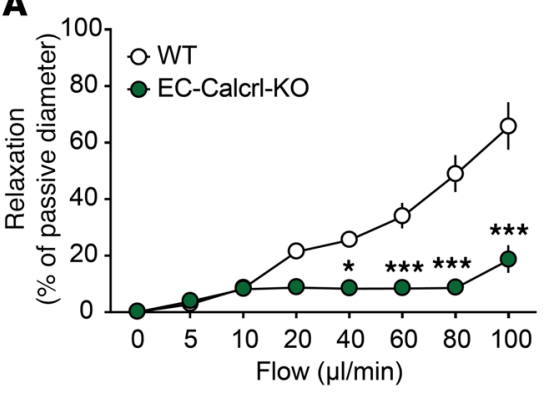

C

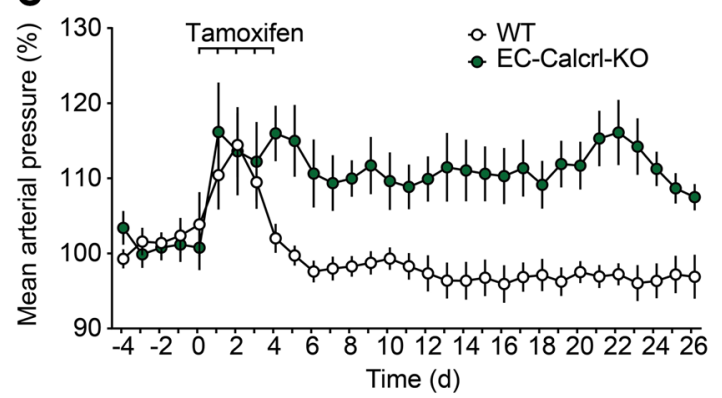

$\square$ Before induction $\square$ After induction

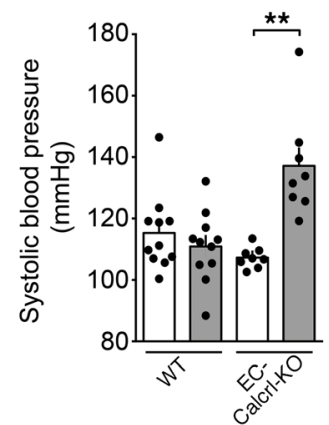

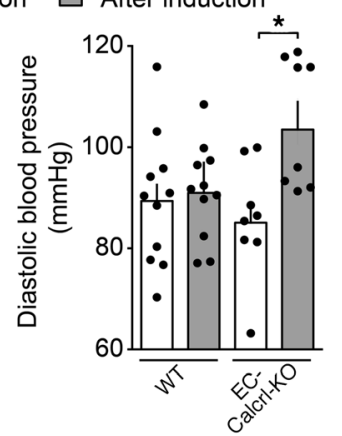
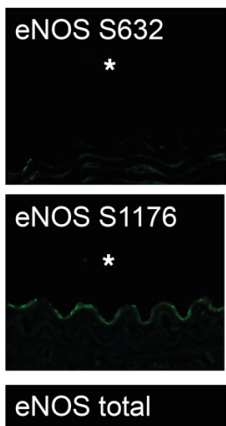
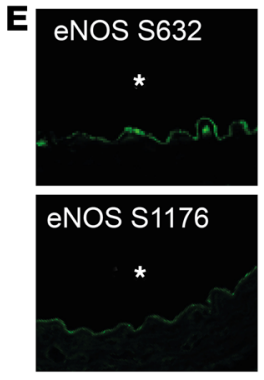

eNOS total
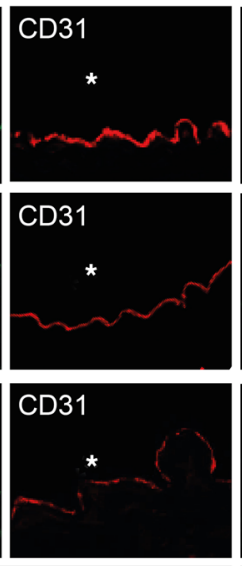

WT

$\mathbf{F}$

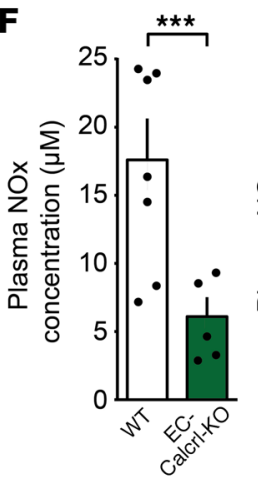

B $\widehat{\bar{\omega}}$
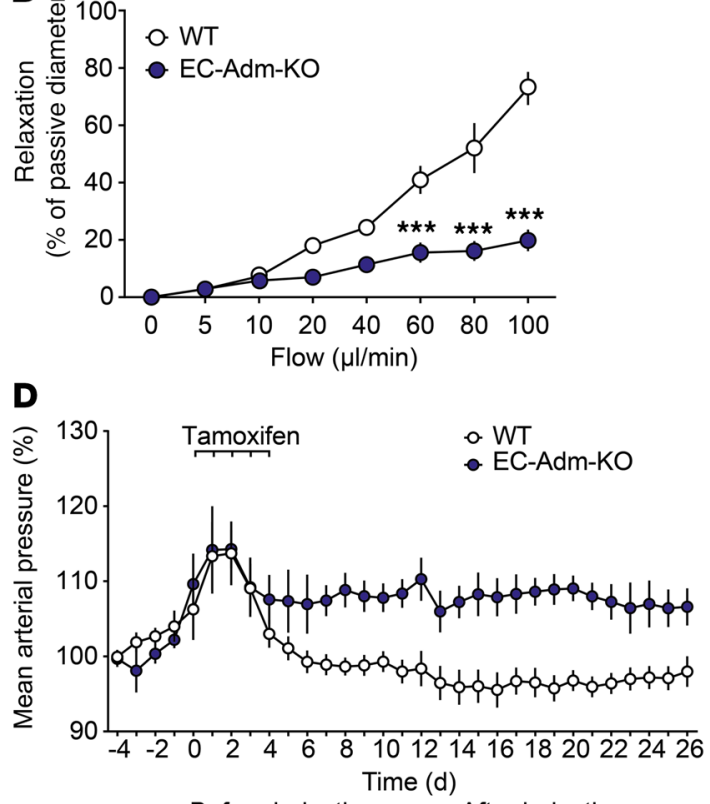

$\square$ Before induction $\square$ After induction
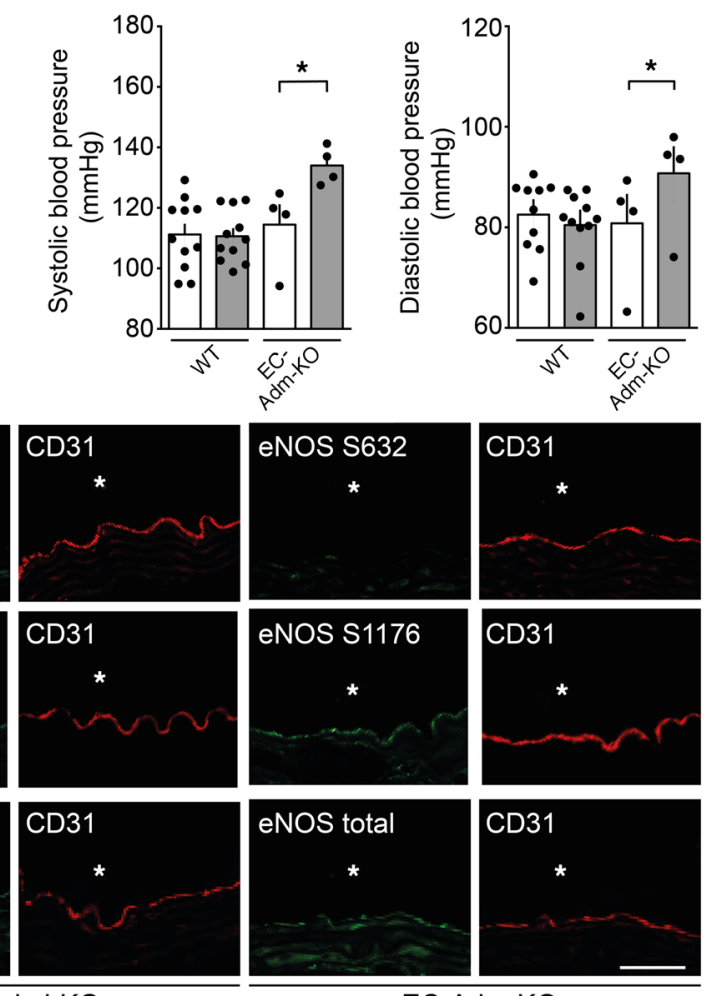

EC-Calcrl-KO

EC-Adm-KO
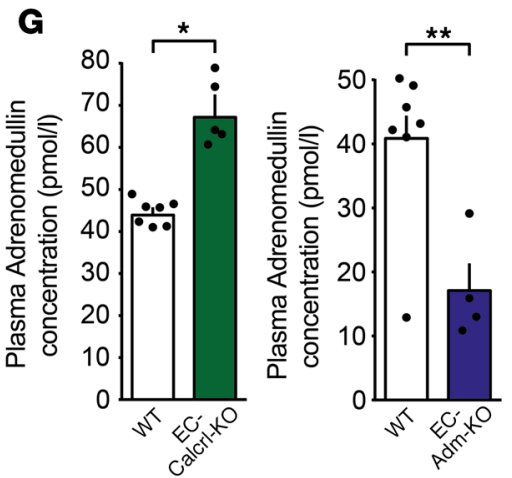
Figure 7. Endothelial adrenomedullin and CALCRL control vascular tone and blood pressure. ( $\mathbf{A}$ and $\mathbf{B}$ ) Effect of increasing perfusion flow on the diameter of mesenteric arteries precontracted with $100 \mathrm{nM} \mathrm{U} 46619$ from EC-Calcrl-KO $(n=8)$ and WT animals $(n=7)$ as well as EC-Adm-KO $(n=6)$ and corresponding WT mice $(n=6)$. (C and D) Blood pressure in WT $(n=11$; C and D), EC-Calcrl-KO mice $(n=8 ; \mathrm{C})$, and EC-Adm-KO animals $(n=4 ; \mathbf{D})$ before, during, and after induction of tamoxifen. Average blood pressure 3 days before induction was set to $100 \%$. Bar diagrams show systolic and diastolic arterial blood pressure 3 days before tamoxifen treatment and during 3 days within the second week after induction. (E) Immuno-confocal microscopy images of aortae isolated from WT or EC- $-\alpha_{s}-\mathrm{KO}$ mice stained with antibodies directed against eNOS, phosphorylated eNOS (S632 or S1176, green), and the endothelial marker CD31 (red). Shown is 1 of 2 independent experiments. Scale bar: $25 \mu \mathrm{m}$. (F and $\mathbf{G}$ ) Plasma nitrite/nitrate (F) and adrenomedullin levels (G) in WT $(n=7)$ and EC-Calcrl-KO animals ( $n$ $=5)$ and EC-Adm-KO mice $(n=4)$ measured 10 days after induction. Data presented are the mean $\pm \mathrm{SEM} ;{ }^{*} P \leq 0.05,{ }^{* *} P \leq 0.01$, ${ }^{* *} P \leq 0.001,2$-way ANOVA with Bonferroni's post hoc test (A and $\mathbf{B}$ ), paired 2-tailed Student's $t$ test (C and $\mathbf{D})$, or unpaired Student's $t$ test (F and $\mathbf{G})$.

ing $3 \mathrm{ml}$ of medium. Shear stress was calculated with the following formula (assuming a Reynolds number of $<<1$ ): $\tau=\eta \times 2 \pi \times n / 0.044$ ( $\tau$, shear stress; $\eta$, viscosity; $n$, rotational speed).

Western blotting. Cells and isolated mesenteric arteries were lysed in radioimmunoprecipitation assay (RIPA) buffer containing $150 \mathrm{mM}$ $\mathrm{NaCl}, 50$ mM Tris-HCl (pH 7.4), 5 mM EDTA, 0.1\% (wt/vol) SDS, 0.5\% sodium deoxycholate, and $1 \%$ Triton X-100 as well as protease inhibitors $[10 \mathrm{mg} / \mathrm{ml}$ leupeptin, pepstatin A, 4-(2-aminoethyl) benzenesulfonyl-fluoride, and aprotinin] and phosphatase inhibitors (PhosSTOP, Roche). Total cell lysates were separated by SDS-PAGE. Protein was then transferred onto nitrocellulose membranes, followed by overnight incubation with primary antibodies. Membranes were incubated with HRP-conjugated secondary antibodies (Cell Signaling Technology) for 1 hour at room temperature and were developed using the ECL detection system (Thermo Scientific Pierce, Life Technologies). In cases in which protein phosphorylation was analyzed using phospho-site-specific antibodies, membranes were first developed with anti-phospho-sitespecific antibodies. After evaluation, antibody dissociation from the membrane was induced using Restore PLUS Western Blot Stripping Buffer (Thermo Fisher Scientific) according to the manufacturer's instructions, and membranes were then reprobed with antibodies recognizing the corresponding protein. Protein band intensities were analyzed by ImageJ software (NIH). Intensity values of bands representing phosphorylated sites of proteins were normalized to the intensity of the band representing total protein.

Immunohistochemical analyses. Mice were sacrificed by $\mathrm{CO}_{2}$ inhalation, and the chest cavity was opened for perfusion with $4^{\circ} \mathrm{C}$ PBS. The aorta was removed and fixed in $4 \%$ PFA at room temperature for 20 minutes. After fixation, adventitial fat was gently peeled away, and aortae were washed 3 times with PBS and incubated in PBS containing 3\% BSA and $0.2 \%$ Triton $\mathrm{X}-100$ for 2 hours. Samples were randomly divided into 3 groups and were incubated overnight at $4^{\circ} \mathrm{C}$ in the same buffer and anti-CD31 antibody (1:100) together with anti-phospho-eNOS S632 antibody, anti-phospho-eNOS S1176 antibody, or eNOS antibody (1:100). After overnight incubation, samples were washed in PBS and incubated with the appropriate Alexa Fluor 564-conjugated antibody and Alexa Fluor 488-conjugated secondary antibody (1:200; Molecular Probes) as well as with DAPI (1:1000) for 1 hour, avoiding exposure to light. Samples were mounted in Aqua-Poly/Mount (Polyscience) overnight. Tissues were imaged using a high-resolution inverted fluorescence microscope platform (Zeiss AxioObserver Z1).

Lentiviral infection of cells. To generate endothelial cells expressing the WT or mutant (S633A; S1177A) form of eNOS, the lentiviral pLVXIRES-ZsGreen1 expression vector (Clontech) containing the sequence of WT or mutant human NOS3 was transfected into HEK293T cells along with envelope plasmid pMD2.G and packaging plasmid psPAX2. The empty vector pLVX-IRES-ZsGreen1 served as control. Following 48 hours of incubation, supernatant was collected and filtered through a $0.45-\mu \mathrm{m}$ low-protein binding Durapore membrane (Millex). HAECs were previously transfected with siRNA against human eNOS using Opti-MEM and Lipofectamine RNAiMAX (Invitrogen). Cells were transduced for 24 hours with the lentiviral vector, followed by a complete medium change. After 48 hours, cells were used for further analyses.

Determination of intracellular cAMP levels. Endothelial cells were plated on culture glass of the BioTech-Flow viscometer chambers. Following laminar shear stress application or treatment with isoproterenol or forskolin, cells were lysed in $0.1 \mathrm{M} \mathrm{HCl}$ supplemented with $0.1 \%$ Triton $\mathrm{X}-100$ and incubated for 10 minutes in room temperature. Lysates were collected and centrifuged $(600 \mathrm{~g}$ for 10 minutes at room temperature) to pellet cellular debris. Intracellular cAMP values were measured using a Direct cAMP ELISA kit (catalog ADI-900-066, Enzo Life Sciences Inc.) following the manufacturer's instructions.

Determination of nitrate and nitrite (NOx) levels. For plasma NOx assays, blood collected by venous puncture was immediately centrifuged at $6800 \mathrm{~g}$ for 5 minutes at $4^{\circ} \mathrm{C}$, and $10 \mu$ plasma was used for NOx measurement. To determine flow-induced NOx release from endothelial cells, $8 \times 10^{3}$ cells $/ \mathrm{cm}^{2}$ were seeded in a flow chamber (MOS Technologies). After reaching confluence, cells were kept under static conditions or were exposed to fluid shear stress using the BioTech-Flow System for up to 30 minutes. One hundred microliters flow medium at different time points was taken, and $10 \mu \mathrm{l}$ was used for the NOx measurement. NOx levels in mouse plasma or cell supernatants were determined using a nitrate/nitrite fluorometric assay kit from Cayman (catalog 780051) according to the manufacturer's instructions. In some experiments, nitrite levels were determined in parallel with an alternative assay system using chemiluminescence (Sievers 280i nitric oxide analyzer). This ozone-based chemiluminescent reaction measures NO directly with very high specificity (66).

Determination of adrenomedullin levels. Supernatants were collected from endothelial cells grown in BioTech-Flow chambers before and after exposure to flow. Samples were transferred to precooled tubes, and cellular debris was removed by centrifugation $(20,000 \mathrm{~g})$ for 5 minutes at $4^{\circ} \mathrm{C}$. Plasma adrenomedullin was measured from blood samples collected by venous puncture of WT control or tissue-specific KO mice, and centrifuged at $6800 \mathrm{~g}$ for 5 minutes at $4^{\circ} \mathrm{C}$. Adrenomedullin concentration was determined with the bovine adrenomedullin ELISA Kit from BIOZOL Diagnostica Vertrieb GmbH (catalog LS-F6083-1) following the manufacturer's instructions.

Animal models. All mice were backcrossed onto a C57BL/6N background at least 8-10 times, and experiments were performed with littermates as controls. Male and female animals (8-12 weeks old) were used unless stated otherwise. Mice were housed under a 12-hour light/12-hour dark cycle, with free access to food and water and under specific pathogen-free conditions unless stated otherwise. The generation of inducible endothelium-specific Cre transgenic mice (Tek-CreER $\left.{ }^{T 2}\right)$ and of floxed $G \alpha_{s}$ mice $\left(G n a s^{t}\right)$ was described previously $(67,68)$. In the Tek-CreER ${ }^{T 2}$ 


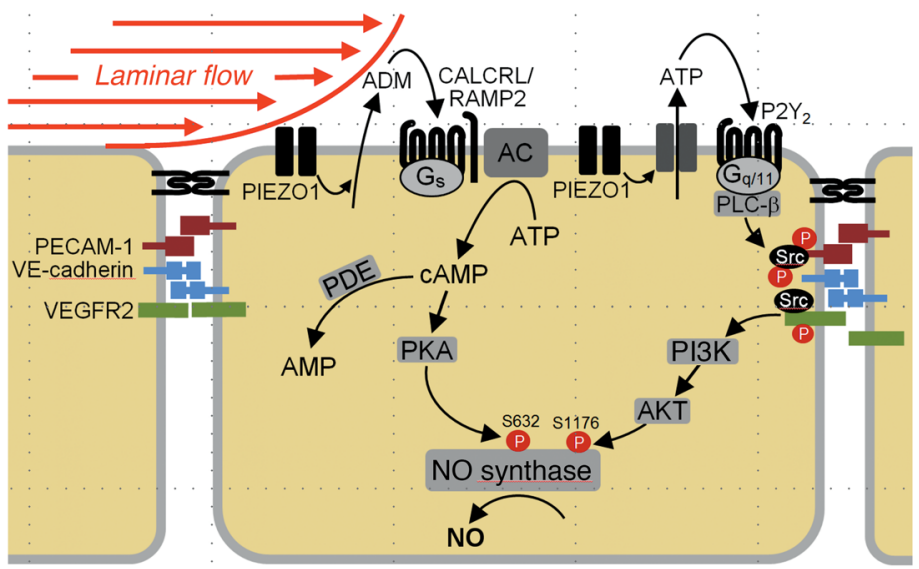

Figure 8. Model of the role of the adrenomedullin receptor and its downstream signaling pathway in flow-induced phosphorylation of eNOS. Laminar flow activates the mechanosensitive cation channel PIEZ01, resulting in the release of adrenomedullin (ADM), which in an autocrine or paracrine fashion activates its receptor consisting of CALCRL and RAMP2, which then through activation of the heterotrimeric $G$ protein $G_{5}$ results in the stimulatory regulation of adenylyl cyclase (AC). The increase in cAMP levels leads to activation of protein kinase $A$ (PKA), which phosphorylates murine eNOS at serine 632, resulting in the activation of the enzyme and increased NO formation. This pathway acts synergistically with $G_{q} / G_{11}$-mediated activation of the mechanosensitive complex consisting of PECAM-1, VE-cadherin, and VEGFR2, which phosphorylates eNOS at serine 1176 via PI3K and AKT. The latter pathway is also induced by laminar flow through PIEZO1 activation and ATP release acting on the $G_{q} / G_{11}$-coupled purinergic $P 2 Y_{2}$ receptor. $P D E$, phosphodiesterase. line, Tie2-driven Cre is activated only in the adult organism, and no recombination in cells of the hematopoietic system was observed (ref. 67 and Supplemental Figure 7). Embryonic stem (ES) cells heterozygous for

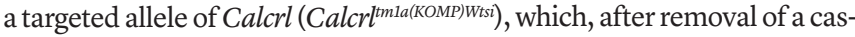
sette flanked by FRT sites, allows for Cre-mediated recombination, were purchased from the KOMP Repository (Knockout Mouse Project, Davis, California, USA). ES cell clones were injected into C57BL/6 blastocysts and were transferred to pseudopregnantfemales. Chimeric offspring were bred with C57BL/6 mice to produce heterozygous animals. The germline transmission was confirmed in the $\mathrm{F}_{1}$ generation using a PCR genotyping strategy. In order to remove the selection cassette, mice were crossed to Flp-deleter mice (69) and were subsequently crossed with Tek-CreER ${ }^{T 2}$ mice to obtain animals with inducible endothelium-specific deficiency. Mice with a conditional knockout allele for the adrenomedullin gene have been previously described (70). These animals were bred with Tek$\mathrm{CreER}^{T 2}$ mice to obtain Tek-CreER ${ }^{T 2} ; A d m^{f / / l l}$ (EC-Adm-KO) mice. Inducible endothelium-specific PIEZO1-deficient mice (Tek-CreER ${ }^{T 2}$;Piezo1 ${ }^{\text {fl/fl }}$ [EC-Piezo1-KO]) have been described before (16).

Telemetric blood pressure measurements. Measurements were performed in conscious, unrestrained mice with a radiotelemetry system (PA-C10, Data Sciences International) as described previously (17). Mice were caged with blinded identity and in random order.

Myography. Adult male mice were transcardially perfused with 10 $\mathrm{ml}$ heparinized Krebs buffer (10 IU/ml) under deep anesthesia. The aorta and the mesenteric arterial bed were removed and placed in ice-cold Krebs buffer (118.1 mM NaCl, $4.8 \mathrm{mM} \mathrm{KCl}, 2.5 \mathrm{mM} \mathrm{CaCl}_{2}, 1.2$ $\mathrm{mM} \mathrm{MgSO}_{4}, 1.2 \mathrm{mM} \mathrm{KH}_{2} \mathrm{PO}_{4}, 25 \mathrm{mM} \mathrm{NaHCO}_{3}, 9.3 \mathrm{mM}$ glucose, and 0.026 mM EDTA, pH 7.4, supplemented with 1\% BSA). Thoracic aortae were cut into segments and were placed on stainless steel vessel holders $(200 \mu \mathrm{m}$ in diameter) in a myograph (610M multiwire myograph system, Danish Myo Technology A/S). Experimental protocol was described previously $(17,71)$. Mice used for the experiments were caged with blinded identity and in random order.

Tissue digestion and cell sorting. In order to validate the effective tissue-specific loss of the gene of interest, lungs of induced-knockout and WT mice were isolated, dissected, and enzymatically digested for 120 minutes while being shaken at $37^{\circ} \mathrm{C}$ in a digestion mix containing collagenase II $(2 \mathrm{mg} / \mathrm{ml}$; Worthington), elastase I $(0.04 \mathrm{mg} / \mathrm{ml}$; Sigma-Aldrich), DNase I ( $5 \mathrm{U} / \mathrm{ml}$; New England BioLabs Inc.), and dispase II (1.2 U/ml; Sigma-Aldrich). Cell suspensions were filtered through $70-\mu \mathrm{m}$ and $40-\mu \mathrm{m}$ cell strainers followed by washing with PBS. Endo- thelial cells were labeled with antibodies directed against CD31 (Serotec MCA2388PE) and sorted on a JSAN cell sorter (Bay Biosciences).

Expression analysis. Total RNA was isolated using the RNeasy Micro Kit (Qiagen). Quality control of samples was carried out using a Nanodrop ND-100 Spectrophotometer. RNA was reversetranscribed using the ProtoScript II cDNA synthesis kit (New England BioLabs Inc.) according to the manufacturer's instructions. Reverse transcriptase PCR reactions were carried out using TaqMan Probe Master (Roche). Primers were designed with the online tool provided by Roche, and quantification was performed using the LightCycler 480 Probe Master System (Roche). Relative expression levels were obtained after normalization with GAPDH values for human and bovine and $18 \mathrm{~S}$ for murine samples.

Quantitative PCR primer sequences. The quantitative PCR (qPCR) primer sequences (species: Bos bovis) were as follows: ADM: 5-ACCGCCAGAGTTTGAACAAC-3 (forward primer), 5-ATGTCCCGAAGCGACAAC-3 (reverse primer); ADM2: 5-GCTCGCAGCCTCAGTACC-3 (forward primer), 5-AGGCTGGCACTCTTCTTGG-3 (reverse primer); CALCA: 5-ATTCTCTGGCATGGGCTTC-3 (forward primer), 5-CCAAGCTGTTGGCTACGTC-3 (reverse primer); CALCRL: 5-TTTACTGTAACAGAACCTGGGATG-3 (forward primer), 5-TTGAAAGTAATCGGGGCAGT-3 (reverse primer); GAPDH: 5-TCACCAGGGCTGCTTTTAAT-3 (forward primer), 5-GAAGGTCAATGAAGGGGTCA-3 (reverse primer); GNAS: 5-GCGCACCATCTCTGTGATT-3 (forward primer), 5-CTTCGATTTTCCAGCAAGGA-3 (reverse primer); GPR146: 5-ACCATGCCCGACGTCTAC-3 (forward primer), 5-CAGGGCGCAGAGTAGCAG-3 (reverse primer); PIEZO1: 5-TCAATGCTAACCACGACTGG-3 (forward primer), 5-GGTCACCGTGTAGCAGAGC-3 (reverse primer); RAMP1: 5-GATGCCAGCCAGAGTGGT-3 (forward primer), 5-CCCAGCAAGACCAACAGC-3 (reverse primer); RAMP2: 5-CTTGGAAAAGGGTGCAGAAG-3 (forward primer), 5-GGCAAAGTGGATGTTGTGAG-3 (reverse primer); RAMP3: 5-ACGTGGTGGGTTGCTACTG-3 (forward primer), 5-TGGAAGAACTGCCTGTGGA-3 (reverse primer). The qPCR primer sequences (species: Mus musculus) were as follows: ADM2: 5-GGAAGTCTCGTCGTCATGC-3 (forward primer), 5-CTGAGGCAGATGAACCATAGC-3 (reverse primer); CALCRL: 5-TAGGACCTGGGACGGATG-3 (forward primer), 5-TCCTGAAAATAGTCAGGGCAGT-3 (reverse primer); RN18S-RS5.1: 5-GCAATTATTCCCCATGAACG-3 (forward primer), 5-TGTACAAAGGGCAGGGACTTA-3 (reverse primer). 
Proteomic and phosphoproteomic analysis. Cells were lysed in RIPA buffer containing $150 \mathrm{mM} \mathrm{NaCl}, 50 \mathrm{mM}$ Tris- $\mathrm{HCl}(\mathrm{pH} 7.4), 5 \mathrm{mM}$ EDTA, 0.1\% (wt/vol) SDS, 0.5\% sodium deoxycholate, and 1\% Triton $\mathrm{X}-100$ as well as protease inhibitors $[10 \mathrm{mg} / \mathrm{ml}$ leupeptin, pepstatin A, 4-(2-aminoethyl) benzenesulfonyl-fluoride, and aprotinin] and phosphatase inhibitors (PhosSTOP, Roche), and cell debris was removed by centrifugation at $16,000 \mathrm{~g}$ for 10 minutes at $4^{\circ} \mathrm{C}$. The concentration of solubilized proteins in the supernatant was determined using the colorimetric 660-nm protein assay (Pierce). Solubilized proteins were precipitated by 4 volumes of $100 \%$ acetone at $-20^{\circ} \mathrm{C}$ overnight. Subsequently, samples were pelleted at 10,000 $\mathrm{g}$ for 10 minutes and pellets washed with $90 \%$ acetone. Samples were dried to remove acetone completely and dissolved in urea buffer (6 M urea, $2 \mathrm{M}$ thiourea, 10 mM HEPES, pH 8.0). Enzymatic peptidolysis was performed by in-solution digestion. Following digestion, the sample was acidified to a final concentration of $0.5 \%$ formic acid (FA) and $2.5 \%$ trifluoroacetic acid (TFA) before reverse-phase extraction using OLIGO R3 resin (Thermo Fisher Scientific). Recovered peptides were dissolved in 50 $\mathrm{mM}$ triethyl ammonium bicarbonate, and peptide concentration was determined using the Fluorimetric Peptide Assay (Pierce). Samples containing an equal amount of peptides $(60 \mu \mathrm{g}$ of peptides per condition and replicate, WT and $\mathrm{G}_{\mathrm{s}}$-knockdown, combined with and without flow, respectively) were subjected to tandem mass tag (TMT), in-solution labeling (Thermo Fisher Scientific).

For proteome analysis, $40 \mu \mathrm{g}$ was fractionated into 8 fractions using the high-pH reverse phase kit (Pierce) according to the manufacturer's recommendations, followed by peptide purification and storage on stop-and-go-extraction tips (StageTips) packed in-house using C18 Empore Disks (3M) (72). After elution in solvent B (80\% acetonitrile, $0.1 \% \mathrm{FA}$ ) and evaporation of acetonitrile (ACN), peptides were dissolved in $0.1 \% \mathrm{FA}$ and a third of the volume subjected to liquid chromatography-tandem mass spectrometry (LC-MS/MS) analysis.

For enrichment and fractionation of phosphorylated peptides, $240 \mu \mathrm{g}$ of labeled peptides were subjected to the $\mathrm{TiO}_{2}$-SIMAC-HILIC (TiSH) protocol (73) with the following changes. A total of $0.9 \mathrm{mg}$ of Titansphere 5- $\mu \mathrm{m} \mathrm{TiO}_{2}$ resin (GL Sciences) was used per $100 \mu \mathrm{g}$ of peptides. For the SIMAC phosphopeptide enrichment, samples were reconstituted in $200 \mu \mathrm{l}$ of buffer containing $50 \% \mathrm{ACN}$ and $2 \% \mathrm{TFA}$, and $80 \mu \mathrm{l}$ of PHOS-Select Iron Affinity Gel (Sigma-Aldrich) was used. The monophosphorylated peptide sample was eluted sequentially by the addition of $1 \mu \mathrm{l}$ of $100 \%$ DMSO, followed by $3.7 \mu \mathrm{l}$ of $\mathrm{H}_{2} \mathrm{O}, 0.5 \mu \mathrm{l}$ of $10 \%$ TFA, and $37.8 \mu \mathrm{l}$ of $100 \% \mathrm{ACN}$. The samples were injected onto an in-house-packed TSKgel Amide-80 3- $\mu \mathrm{m}$ HILIC resin $300 \mu \mathrm{m} \times$ $150 \mathrm{~mm}$ capillary HPLC column using an Agilent 1260 Infinity capillary HPLC system. A total number of 11 fractions was collected, dried by vacuum centrifugation, and dissolved in $7 \mu \mathrm{l}$ of $0.1 \%$ FA. Of each fraction, $3 \mu \mathrm{l}$ was subjected to LC-MS/MS analysis.

Peptides were separated using a UHPLC system (EASY-nLC 1000, Thermo Fisher Scientific) and 20-cm, in-house-packed C18 silica columns (1.9- $\mu \mathrm{m} \mathrm{C18}$ beads, Dr. Maisch $\mathrm{GmbH})$ coupled in line to a QExactive HF Orbitrap mass spectrometer (Thermo Fisher Scientific) using an electrospray ionization source. The high-pH reversephase fractionated peptides for proteome analysis were subjected to a linearly increasing gradient concentration of solvent B (80\% ACN, $0.1 \% \mathrm{FA}$ ) over solvent A (0.1\% FA) from $7 \%$ to $25 \%$ for 100 minutes and from $25 \%$ to $60 \%$ for 20 minutes, followed by washing with $95 \%$ of solvent B for 5 minutes and re-equilibration with $5 \%$ of solvent B.
Full MS spectra were acquired in a mass range of 300-1750 m/z with a resolution of 120,000 at $200 \mathrm{~m} / z$. The ion injection target was set to $3 \times 10^{6}$ and the maximum injection time limited to 50 milliseconds. Ions were fragmented by high-energy collision dissociation using a normalized collision energy of 32 and an ion injection target of $1.0 \times$ $10^{5}$ with a maximum injection time of 100 milliseconds. The resulting tandem mass spectra (MS/MS) were acquired with a resolution of 30,000 at $200 \mathrm{~m} / z$ using data-dependent mode with a loop count of 15 (top15). LC-MS/MS analysis of phosphopeptide-enriched samples followed a comparable paradigm with the following modifications: separation in-line to the mass spectrometry was achieved using a linearly increasing gradient concentration of solvent B over solvent A from $10 \%$ to $38 \%$ for 77 minutes and from $38 \%$ to $60 \%$ for 5 minutes, followed by washing with $95 \%$ of solvent B for 5 minutes and re-equilibration with $5 \%$ of solvent B. Full MS spectra were acquired with a resolution of 60,000 at $200 \mathrm{~m} / z$. The ion injection target was set to $3 \times 10^{6}$ and the maximum injection time limited to 20 milliseconds. For fragmentation spectra an ion injection target of $5.0 \times 10^{5}$ with a maximum injection time of 120 milliseconds was used.

MS raw data were processed by MaxQuant (1.6.1.0) (74) using the UniProt bovine database containing 32,231 entries (release 09.02.2018). The following parameters were used for data processing in 2 parameter groups: maximum of 2 missed cleavages, mass tolerance of $4.5 \mathrm{ppm}$ for main search, trypsin as digesting enzyme, carbamidomethylation of cysteines as fixed modification, and 2 parameter groups both using oxidation of methionine and acetylation of the protein N-terminus as variable modifications. For the phosphoproteome-specific parameter group, phosphorylation of serines, threonines, and tyrosines was used as additional variable modifications. For quantification based on TMT labeling, different isotope labels were set as variable modification on the peptide N-termini and lysine residues. Peptides with a minimum of 7 amino acids and at least 1 unique peptide were included in protein identification. Only proteins with at least 2 peptides and at least 1 unique peptide were considered as identified and were used for further data analysis.

RNA-Seq. RNA was isolated from HUVECs and BAECs using the miRNeasy Micro Kit (Qiagen). Samples were treated with on-column DNase digestion (DNase-Free DNase Set, Qiagen), and total RNA and library integrity were verified with LabChip Gx Touch 24 (PerkinElmer). Two micrograms of human total RNA and $4 \mu \mathrm{g}$ of bovine total RNA were used as input for TruSeq Stranded mRNA Library preparation following the Low Sample protocol (Illumina). Sequencing was performed on a NextSeq500 instrument (Illumina) using v2 chemistry, resulting in an average of 25 million reads per library for human and 35 million reads per library for bovine sample with a $75 \mathrm{bp}$ single-end setup. The resulting raw reads were assessed for quality, adapter content, and duplication rates. Only reads between 30 and 150 nucleotides were cleared for further analyses. Trimmed and filtered reads were aligned versus the Ensembl human genome version hg38 (GRCh38) or Ensembl Bos taurus genome version umd3.1 using STAR 2.4.0a with the parameter "--outFilterMismatchNoverLmax 0.1 " to increase the maximum ratio of mismatches to mapped length to $10 \%(75)$. The number of reads aligning to genes was counted with featureCounts 1.4.5-p1 tool from the Subread package (76). Only reads mapping at least partially inside exons were admitted and aggregated per gene. Reads overlapping multiple genes or aligning to multiple regions were excluded. Differentially expressed genes were 
identified using DESeq2 version 1.62 (77). Only genes with a minimum fold change of $\pm 1.5\left(\log _{2} \pm 0.59\right)$, a maximum Benjamini-Hochberg corrected $P$ value of 0.05 , and a minimum combined mean of 5 reads were deemed to be significantly differentially expressed. The Ensembl annotation was enriched with UniProt data (release 06.06.2014) based on Ensembl gene identifiers (Activities at the Universal Protein Resource (UniProt), https://www.ncbi.nlm.nih. gov/pubmed/24253303). Gene expression was further normalized toward transcripts per million (TPM) values to ensure comparability between different species. To accomplish this, the number of nucleotides overlapping an exon were counted per gene. The read count per gene was then divided by the length of summed exons per gene in kilobases (reads per kilobase [RPK]). These values were summed per sample and divided by $1,000,000$, resulting in a scaling factor per sample. Finally, the RPK values of each gene were divided by the scaling factor to obtain TPMs.

Statistics. Statistical analysis was performed using GraphPad Prism software version 6.07 (GraphPad Software Inc.). Values are presented as mean \pm SEM; $n$ represents the number of independent experiments. Statistical analysis between 2 groups was performed with an unpaired 2-tailed Student's $t$ test, while multiple-group comparisons were analyzed with 1-way ANOVA followed by Tukey's post hoc test, unless stated otherwise, and comparisons between multiple groups at different time points were performed using 2-way ANOVA followed by Bonferroni's post hoc test. A $P$ value less than 0.05 was considered to be statistically significant.
Study approval. All procedures involving animal care and use in this study were approved by the local animal ethics committees (Regierungspräsidia Karlsruhe and Darmstadt, Germany).

\section{Author contributions}

AI performed most of the in vitro and in vivo experiments, analyzed and discussed data, and contributed to writing the manuscript. YJJ, JAJ, MS, SW, PTD, AN, and ST conducted experiments and acquired and analyzed data. CK and SG performed RNA sequencing and bioinformatics analysis. MC, AM, and LSW provided genetically modified mouse lines. IF and NW discussed data. AMS and JG performed phosphoproteomic analysis. SO initiated and supervised the study, discussed data, and wrote the manuscript. All authors read and commented on the manuscript.

\section{Acknowledgments}

We thank Svea Hümmer for secretarial help and Karin Jäcklein, Anne Konzer, and Sylvia Jeratsch for expert technical support. This work was supported by the Collaborative Research Center 834 of the German Research Foundation.

Address correspondence to: Stefan Offermanns, Max Planck Institute for Heart and Lung Research, Ludwigstrasse 43, $61231 \mathrm{Bad}$ Nauheim, Germany. Phone: 49.0.6032.705.1201; Email: stefan. offermanns@mpi-bn.mpg.de.
1. Baeyens N, Bandyopadhyay C, Coon BG, Yun S, Schwartz MA. Endothelial fluid shear stress sensing in vascular health and disease. J Clin Invest. 2016;126(3):821-828.

2. Félétou M, Köhler R, Vanhoutte PM. Endotheliumderived vasoactive factors and hypertension: possible roles in pathogenesis and as treatment targets. Curr Hypertens Rep. 2010;12(4):267-275.

3. Fleming I. Molecular mechanisms underlying the activation of eNOS. Pflugers Arch. 2010;459(6):793-806.

4. Balligand JL, Feron O, Dessy C. eNOS activation by physical forces: from short-term regulation of contraction to chronic remodeling of cardiovascular tissues. Physiol Rev. 2009;89(2):481-534.

5. Garcia V, Sessa WC. Endothelial NOS: perspective and recent developments. Br J Pharmacol. 2019;176(2):189-196.

6. Michel T, Vanhoutte PM. Cellular signaling and NO production. Pflugers Arch. 2010;459(6):807-816.

7. Kuchan MJ, Jo H, Frangos JA. Role of G proteins in shear stress-mediated nitric oxide production by endothelial cells. Am J Physiol. 1994;267(3 pt 1):C753-C758.

8. Fulton D, et al. Regulation of endotheliumderived nitric oxide production by the protein kinase Akt. Nature. 1999;399(6736):597-601.

9. Dimmeler S, Fleming I, Fisslthaler B, Hermann C, Busse R, Zeiher AM. Activation of nitric oxide synthase in endothelial cells by Akt-dependent phosphorylation. Nature. 1999;399(6736):601-605.

10. Tzima E, et al. A mechanosensory complex that mediates the endothelial cell response to fluid shear stress. Nature. 2005;437(7057):426-431.

11. Conway D, Schwartz MA. Lessons from the endo- thelial junctional mechanosensory complex. F1000 Biol Rep. 2012;4:1.

12. Coon BG, et al. Intramembrane binding of VE-cadherin to VEGFR2 and VEGFR3 assembles the endothelial mechanosensory complex. JCell Biol. 2015;208(7):975-986.

13. Baeyens N, Schwartz MA. Biomechanics of vascular mechanosensation and remodeling. Mol Biol Cell. 2016;27(1):7-11.

14. Ranade SS, et al. Piezo1, a mechanically activated ion channel, is required for vascular development in mice. Proc Natl Acad Sci U S A. 2014;111(28):10347-10352.

15. Li J, et al. Piezo1 integration of vascular architecture with physiological force. Nature. 2014;515(7526):279-282.

16. Wang S, Chennupati R, Kaur H, Iring A, Wettschureck N, Offermanns S. Endothelial cation channel PIEZO1 controls blood pressure by mediating flow-induced ATP release. J Clin Invest. 2016;126(12):4527-4536.

17. Wang S, et al. P2Y and $G q / G_{11}$ control blood pressure by mediating endothelial mechanotransduction. JClin Invest. 2015;125(8):3077-3086.

18. Qian J, Fulton D. Post-translational regulation of endothelial nitric oxide synthase in vascular endothelium. Front Physiol. 2013;4:347.

19. Michell BJ, et al. Identification of regulatory sites of phosphorylation of the bovine endothelial nitric-oxide synthase at serine 617 and serine 635. J Biol Chem. 2002;277(44):42344-42351.

20. Boo YC, et al. Endothelial NO synthase phosphorylated at SER635 produces NO without requiring intracellular calcium increase. Free Radic Biol Med. 2003;35(7):729-741.
21. Bauer PM, et al. Compensatory phosphorylation and protein-protein interactions revealed by loss of function and gain of function mutants of multiple serine phosphorylation sites in endothelial nitric-oxide synthase. J Biol Chem. 2003;278(17):14841-14849.

22. Boo YC, Kim HJ, Song H, Fulton D, Sessa W, Jo H. Coordinated regulation of endothelial nitric oxide synthase activity by phosphorylation and subcellular localization. Free Radic Biol Med. 2006;41(1):144-153.

23. Butt E, et al. Endothelial nitric-oxide synthase (type III) is activated and becomes calcium independent upon phosphorylation by cyclic nucleotide-dependent protein kinases. J Biol Chem. 2000;275(7):5179-5187.

24. Boo YC, et al. Shear stress stimulates phosphorylation of eNOS at Ser(635) by a protein kinase A-dependent mechanism. Am J Physiol Heart Circ Physiol. 2002;283(5):H1819-H1828.

25. Cheng HC, et al. A potent synthetic peptide inhibitor of the cAMP-dependent protein kinase. JBiol Chem. 1986;261(3):989-992.

26. Narayana N, Cox S, Shaltiel S, Taylor SS, Xuong N. Crystal structure of a polyhistidine-tagged recombinant catalytic subunit of cAMP-dependent protein kinase complexed with the peptide inhibitor PKI(5-24) and adenosine. Biochemistry. 1997;36(15):4438-4448.

27. Taylor SS, Ilouz R, Zhang P, Kornev AP. Assembly of allosteric macromolecular switches: lessons from PKA. Nat Rev Mol Cell Biol. 2012;13(10):646-658.

28. Reich KM, Gay CV, Frangos JA. Fluid shear stress as a mediator of osteoblast cyclic adenos- 
ine monophosphate production. J Cell Physiol. 1990;143(1):100-104

29. Simonds WF. G protein regulation of adenylate cyclase. Trends Pharmacol Sci. 1999;20(2):66-73.

30. Wettschureck N, Offermanns S. Mammalian G proteins and their cell type specific functions. Physiol Rev. 2005;85(4):1159-1204.

31. Bevan JA, Laher I. Pressure and flow-dependent vascular tone. FASEB J.1991;5(9):2267-2273.

32. Hahn C, Schwartz MA. Mechanotransduction in vascular physiology and atherogenesis. Nat Rev Mol Cell Biol. 2009;10(1):53-62.

33. Poyner DR, et al. International Union of Pharmacology. XXXII. The mammalian calcitonin gene-related peptides, adrenomedullin, amylin, and calcitonin receptors. Pharmacol Rev. 2002;54(2):233-246.

34. Koyama T, Sakurai T, Kamiyoshi A, IchikawaShindo Y, Kawate H, Shindo T. AdrenomedullinRAMP2 system in vascular endothelial cells. JAtheroscler Thromb. 2015;22(7):647-653.

35. Zhang SY, Xu MJ, Wang X. Adrenomedullin 2/ intermedin: a putative drug candidate for treatment of cardiometabolic diseases. Br JPharmacol.2018;175(8):1230-1240.

36. Zhao Y, Vanhoutte PM, Leung SW. Vascular nitric oxide: beyond eNOS. J Pharmacol Sci. 2015;129(2):83-94.

37. Boo YC, Jo H. Flow-dependent regulation of endothelial nitric oxide synthase: role of protein kinases. Am J Physiol Cell Physiol. 2003;285(3):C499-C508.

38. Boo YC, et al. Shear stress stimulates phosphorylation of endothelial nitric-oxide synthase at Ser1179 by Akt-independent mechanisms: role of protein kinase A. J Biol Chem. 2002;277(5):3388-3396.

39. Davies SP, Reddy H, Caivano M, Cohen P. Specificity and mechanism of action of some commonly used protein kinase inhibitors. Biochem $J$. 2000;351(pt 1):95-105.

40. Chachisvilis M, Zhang YL, Frangos JA. G protein-coupled receptors sense fluid shear stress in endothelial cells. Proc Natl Acad Sci U S A. 2006;103(42):15463-15468.

41. Jung B, et al. Flow-regulated endothelial S1P receptor-1 signaling sustains vascular development. Dev Cell. 2012;23(3):600-610.

42. Xu J, et al. GPR68 senses flow and is essential for vascular physiology. Cell. 2018;173(3):762-775.e16.

43. Martínez A, Arias J, Bassuk JA, Wu H, Kurlansky $\mathrm{P}$, Adams JA. Adrenomedullin is increased by pulsatile shear stress on the vascular endothelium via periodic acceleration ( $\mathrm{pGz}$ ). Peptides. 2008;29(1):73-78.

44. Chun TH, et al. Shear stress augments expression of C-type natriuretic peptide and adrenomedullin. Hypertension. 1997;29(6):1296-1302.

45. Dekker RJ, et al. Endothelial KLF2 links local arterial shear stress levels to the expression of vascular tone-regulating genes. Am J Pathol. 2005;167(2):609-618.

46. Minamino N, Kikumoto K, Isumi Y. Regulation of adrenomedullin expression and release. Microsc Res Tech. 2002;57(1):28-39.

47. Katoh F, et al. $\mathrm{Ca}(2+)$-dependent cosecretion of adrenomedullin and catecholamines mediated by nicotinic receptors in bovine cultured adrenal medullary cells. FEBS Lett. 1994;348(1):61-64.

48. Birukov KG, et al. Shear stress-mediated cytoskeletal remodeling and cortactin translocation in pulmonary endothelial cells. Am J Respir Cell Mol Biol. 2002;26(4):453-464.

49. Sinha S, et al. Cortactin promotes exosome secretion by controlling branched actin dynamics. J Cell Biol. 2016;214(2):197-213.

50. Sung BH, Weaver AM. Regulation of lysosomal secretion by cortactin drives fibronectin deposition and cell motility. Bioarchitecture. 2011;1(6):257-260.

51. Sung BH, Zhu X, Kaverina I, Weaver AM. Cortactin controls cell motility and lamellipodial dynamics by regulating ECM secretion. Curr Biol. 2011;21(17):1460-1469.

52. Zhao G, Zhang H, Huang Z, Lv L, Yan F. Cortactin and Exo70 mediated invasion of hepatoma carcinoma cells by MMP-9 secretion. Mol Biol Rep. 2016;43(5):407-414.

53. García Ponce A, et al. Loss of cortactin causes endothelial barrier dysfunction via disturbed adrenomedullin secretion and actomyosin contractility. Sci Rep. 2016;6:29003.

54. Kato J, Tsuruda T, Kita T, Kitamura K, Eto T. Adrenomedullin: a protective factor for blood vessels. Arterioscler Thromb Vasc Biol. 2005;25(12):2480-2487.

55. Hinson JP, Kapas S, Smith DM. Adrenomedullin, a multifunctional regulatory peptide. Endocr Rev 2000;21(2):138-167.

56. Ishimitsu T, et al. Plasma levels of adrenomedullin, a newly identified hypotensive peptide, in patients with hypertension and renal failure. J Clin Invest. 1994;94(5):2158-2161.

57. Hu W, Zhou PH, Zhang XB, Xu CG, Wang W. Plasma concentrations of adrenomedullin and natriuretic peptides in patients with essential hypertension. Exp Ther Med. 2015;9(5):1901-1908

58. Lewis LK, Smith MW, Yandle TG, Richards AM, Nicholls MG. Adrenomedullin(1-52) measured in human plasma by radioimmunoassay: plasma concentration, adsorption, and storage. Clin Chem. 1998;44(3):571-577.

59. Kohno M, et al. Plasma adrenomedullin concentrations in essential hypertension. Hypertension. 1996;27(1):102-107.

60. Watkins HA, et al. Receptor activity-modifying proteins 2 and 3 generate adrenomedullin receptor subtypes with distinct molecular properties. J Biol Chem. 2016;291(22):11657-11675.

61. Iesato Y, et al. Adrenomedullin-RAMP2 system is crucially involved in retinal angiogenesis. Am J Pathol. 2013;182(6):2380-2390.

62. Tanaka M, et al. The endothelial adrenomedullinRAMP2 system regulates vascular integrity and suppresses tumour metastasis. Cardiovasc Res.
2016;111(4):398-409.

63. Koyama T, et al. Vascular endothelial adrenomedullin-RAMP2 system is essential for vascular integrity and organ homeostasis. Circulation. 2013;127(7):842-853

64. Ochoa-Callejero L, Pozo-Rodrigálvarez A, Martínez-Murillo R, Martínez A. Lack of adrenomedullin in mouse endothelial cells results in defective angiogenesis, enhanced vascular permeability, less metastasis, and more brain damage. Sci Rep. 2016;6:33495.

65. Buschmann MH, Dieterich P, Adams NA, Schnittler HJ. Analysis of flow in a cone-andplate apparatus with respect to spatial and temporal effects on endothelial cells. Biotechnol Bioeng. 2005;89(5):493-502.

66. Hetrick EM, Schoenfisch MH. Analytical chemistry of nitric oxide. Annu Rev Anal Chem (Palo Alto Calif). 2009;2:409-433.

67. Korhonen H, et al. Anaphylactic shock depends on endothelial Gq/G11.J Exp Med. 2009;206(2):411-420.

68. Chen M, et al. Increased glucose tolerance and reduced adiposity in the absence of fasting hypoglycemia in mice with liver-specific Gs alpha deficiency. JClin Invest. 2005;115(11):3217-3227.

69. Farley FW, Soriano P, Steffen LS, Dymecki SM. Widespread recombinase expression using FLPeR (flipper) mice. Genesis. 2000;28(3-4):106-110.

70. Fernández AP, Serrano J, Tessarollo L, Cuttitta F, Martínez A. Lack of adrenomedullin in the mouse brain results in behavioral changes, anxiety, and lower survival under stress conditions. Proc Natl Acad Sci U S A. 2008;105(34):12581-12586.

71. Dancs PT, et al. LPA1 receptor-mediated thromboxane A2 release is responsible for lysophosphatidic acid-induced vascular smooth muscle contraction. FASEB J. 2017;31(4):1547-1555.

72. Rappsilber J, Ishihama Y, Mann M. Stop and go extraction tips for matrix-assisted laser desorption/ionization, nanoelectrospray, and LC/MS sample pretreatment in proteomics. Anal Chem. 2003;75(3):663-670.

73. Engholm-Keller K, Larsen MR. Improving the phosphoproteome coverage for limited sample amounts using $\mathrm{TiO}_{2}$-SIMAC-HILIC (TiSH) phosphopeptide enrichment and fractionation. Methods Mol Biol. 2016;1355:161-177.

74. Cox J, Mann M. MaxQuant enables high peptide identification rates, individualized p.p.b.range mass accuracies and proteome-wide protein quantification. Nat Biotechnol. 2008;26(12):1367-1372.

75. Dobin A, et al. STAR: ultrafast universal RNA-seq aligner. Bioinformatics. 2013;29(1):15-21.

76. Liao Y, Smyth GK, Shi W. featureCounts: an efficient general purpose program for assigning sequence reads to genomic features. Bioinformatics. 2014;30(7):923-930.

77. Love MI, Huber W, Anders S. Moderated estimation of fold change and dispersion for RNA-seq data with DESeq2. Genome Biol. 2014;15(12):550. 